\title{
3 Researc Square

\section{Amplifying the heat shock response ameliorates pathology in mouse and human models of ALS and FTD.}

\section{Mhoriam Ahmed}

University College London

Charlotte Spicer

University College London

Jasmine Harley

The Francis Crick Institute

Nikolaj Petersen

Orphazyme A/S, Copenhagen

\section{Paul Taylor}

St. Jude Children's Research Hospital

\section{Thomas Jensen}

Orphazyme A/S, Copenhagen https://orcid.org/0000-0001-5931-263X

\section{Michael Hanna}

University College London

\section{Rickie Patani}

The Francis Crick Institute https://orcid.org/0000-0002-3825-7675

Linda Greensmith ( $\nabla$ l.greensmith@ucl.ac.uk)

University College London

\section{Article}

Keywords: Amyotrophic lateral sclerosis (ALS), frontotemporal dementia (FTD), heat shock response

Posted Date: January 29th, 2021

DOI: https://doi.org/10.21203/rs.3.rs-152813/v1

License: (c) (1) This work is licensed under a Creative Commons Attribution 4.0 International License. Read Full License 
1 Amplifying the heat shock response ameliorates pathology in mouse and human models of

ALS and FTD

12 ${ }^{4}$ Department of Cell and Molecular Biology, St. Jude Children's Research Hospital, Memphis, TN $13 \quad 38105$

${ }^{*}$ Correspondence to Professor Linda Greensmith at above address

17

18 
ABSTRACT

Amyotrophic lateral sclerosis (ALS) and frontotemporal dementia (FTD) are now widely considered to be part of a disease spectrum with the identification of common pathological features and genetic causes. However, despite these advances, there remains no effective therapy for these conditions. In this study we demonstrate that mice expressing mutant valosin containing protein (VCP) develop an ALS/FTD-like phenotype in the spinal cord and brain, and treatment with arimoclomol, a pharmacological amplifier of the cytoprotective heat shock response ameliorates this phenotype. Moreover, the beneficial effects of arimoclomol are seen in both fibroblasts and iPSC-derived motor neurons from patients. Importantly, we show the pathological changes targeted by arimoclomol in our experimental models are present in post-mortem FTD patient tissue. Together with previous data demonstrating the efficacy of arimoclomol in SOD1-ALS models, our findings suggest that arimoclomol may have therapeutic potential not only in non-SOD1 ALS but also for the treatment of FTD.

Abbreviations:

$\mathrm{ALS}=$ Amyotrophic lateral sclerosis, EDL = Extensor digitorum longus, FTD = Frontotemporal dementia, FMRP = Fragile X mental retardation protein, FUS = Fused in Sarcoma, G3BP = Ras-GAP SH3 domain binding protein, GFAP = Glial fibrillary acid protein, HSF-1 = Heat shock factor-1, HSP = Heat shock protein, HSP70 = Heat shock protein 70, HSR = Heat shock response, IBMPFD = Inclusion body myopathy with Paget's disease and Frontotemporal dementia, iPSC $=$ induced pluripotent stem cell, LC3 $=$ Microtubule-associated protein 1A/1Blight chain 3, MAPT = microtubule -associated protein tau, $\mathrm{MN}=$ Motor neuron, $\mathrm{MSP}=$ Multisystem proteinopathy, $\mathrm{mVCP}=$ mutant valosin containing protein, $\mathrm{PD}=$ Paget's disease, $\mathrm{SBMA}=$ Spinal and bulbar muscular atrophy, SOD = Superoxide dismutase, TDP-43 = TAR DNA binding protein 43, UPS = Ubiquitinproteasome system, $\mathrm{VCP}=$ Valosin containing protein, $\mathrm{wtVCP}=$ wildtype human valosin containing protein, $\mathrm{WT}$

$60=$ Wildtype 


\section{INTRODUCTION}

Although Amyotrophic Lateral Sclerosis (ALS) and Frontotemporal Dementia (FTD) have been traditionally categorised as distinct neurodegenerative disorders, it is now widely accepted that they form part of a degenerative disease continuum ${ }^{1}$, with overlapping genetic, clinical and pathological features. A major genetic link between familial ALS and FTD was identified with the discovery of the GGGGCC pathogenic repeat expansions in the C9orf72 gene, which accounts for approximately $25 \%$ of familial FTD and $40 \%$ of familial ALS cases ${ }^{2,3}$. Other, less frequently occurring mutations including those in TARDBP, SQSTM1 and VCP genes ${ }^{4}$ have also been identified, although the precise mechanism underlying the resulting pathology has yet to be fully determined for any of these mutations.

Mutations in the Valosin Containing Protein (VCP/p97) gene on chromosome 9p13-p12 are associated with both familial FTD (<1\%) and familial ALS (1-2\%) ${ }^{5,6}$. Expression of mutant VCP leads to a multisystem inherited pleiotropic disorder that can affect muscle, bone, and the nervous system, now referred to as multisystem proteinopathy (MSP), but previously referred to by the acronym IBMPFD (Inclusion Body Myopathy (IBM) with Paget's disease of bone (PDB) and FTD). Patients with MSP present with a combination of clinical and pathological symptoms that includes ALS as well as FTD, the muscle disorder, IBM and PDB. Whilst ALS, FTD and IBM pathology are distinct in each tissue and can exist independently, they share common pathological hallmarks which are indicative of protein dyshomeostasis, with the presence of ubiquitin-positive inclusion bodies, p62 aggregation and mislocalisation of the RNA-binding protein, TDP-43, observed in all three disorders ${ }^{7,8}$.

VCP is a ubiquitously expressed protein which forms a monohexamer containing three main domains: The $\mathrm{N}$ domain, mainly associated with substrate binding, the D1 domain for oligomerisation and ATP hydrolysis and the D2 domain which also undertakes ATPase activity ${ }^{9}$. VCP associates with a large range of co-factors to bring about diverse cellular functions ranging from progression of the cell cycle to membrane fusion ${ }^{9}$. As a segregase, powered by ATP hydrolysis, VCP extracts and unfolds substrate proteins ubiquitinated for degradation. VCP is also linked to maturation of autophagosomes which are crucial for autophagy to take place ${ }^{10}$. This protein is therefore heavily involved in the two major protein degradation mechanisms of the cell: autophagy and the ubiquitin-proteasome system (UPS) ${ }^{11,12}$. It therefore follows that mutations in VCP may alter cellular protein homeostasis by dysregulating these important housekeeping systems. This in turn is likely to increase the aberrant protein load in a cell leading to proteinaceous aggregate formation, potentially accounting for the degenerative pathology seen in the spinal cord, brain and muscle of MSP patients with mutant VCP and linking protein dyshomeostasis as a pathomechanism in other ALS/FTD cases. 
110 Restoration of protein homeostasis is therefore a potential therapeutic approach to ameliorate 111 pathology in MSP and ALS/FTD. In this study, we examined the possibility that targeting the 112 endogenous protein handling mechanism known as the Heat Shock Response (HSR) may be a 113 successful strategy to restore protein homeostasis in MSP and ALS/FTD. The HSR is a ubiquitous, 114 cytoprotective signalling pathway that is triggered under conditions of acute or chronic stress, leading 115 to activation of the transcription factor Heat Shock Factor 1 (HSF-1) ${ }^{13}$, and subsequent upregulation 116 of the family of molecular chaperones know as heat shock proteins (HSPs). HSPs sequester 117 hydrophobic regions of misfolded proteins to prevent protein-protein interaction and subsequent 118 aggregation. HSPs are also involved in regulating lysosomal function and autophagy by delivering 119 aberrant proteins to the surface of lysosomes and enabling autophagic degradation ${ }^{14}$. Indeed HSF-1 120 itself has been shown to regulate autophagy in the cell, such as by altering SQSTM1/p62 121 phosphorylation and at the transcriptional level by upregulating Atg genes required for autophagy ${ }^{15}$,

$122{ }^{16}$. In addition, HSP70, a major player in the chaperone system has been shown to stabilise lysosomal 123 integrity ${ }^{17-19}$. Therefore, upregulation of HSPs expression in protein-misfolding diseases such as MSP 124 and ALS/FTD may be an effective approach to restore the proteostasis impaired by aberrant proteins 125 and improve cellular health.

127 We have previously tested the potential therapeutic effects of augmenting the HSR using a 128 pharmacological amplifier of called arimoclomol. Treatment with arimoclomol improved both 129 neuropathological and functional deficits in mouse models of two motor neuron diseases - the 130 SOD1 ${ }^{\text {G93A }}$ mouse model of ALS ${ }^{20-22}$, and in the AR100 mouse model of Spinal and Bulbar Muscular 131 Atrophy (SBMA) ${ }^{23}$. Arimoclomol acts like a smart-drug, prolonging the activation of HSF-1, but only 132 in cells in which the HSR has already been activated in response to stress ${ }^{20,24,25}$. The unique ability 133 of arimoclomol to amplify rather than activate the HSR, makes it a particularly attractive therapeutic 134 compound as it avoids the off-target side effects of widespread, non-specific activation of the HSR in 135 multiple cell types, including those not affected in disease and not under stress; this is a major 136 drawback of other approaches to upregulate the HSR.

138 Furthermore, we have also shown that treatment with arimoclomol ameliorates skeletal muscle 139 pathology in models of IBM, including in mice expressing humanised VCP with the dominant mutation 140 A232E (mVCP), which manifest the characteristic pathological features of IBM ${ }^{26,27}$. Treatment of 141 mVCP mice with arimoclomol not only reduced protein aggregation in muscle fibres of mVCP mice, 142 but also reduced TDP-43 mislocalisation, myofibre atrophy and degeneration. Importantly, these 143 improvements in IBM-like pathology in muscles of mVCP mice were reflected by significant 144 improvements in muscle function, including increased force generation. These beneficial effects of 145 arimoclomol in mVCP mice are likely to result, at least in part, from an increase in the expression of 146 HSPs, as a two-fold increase in the expression of HSP70 was observed in muscles of treated mVCP 
mice compared to that of untreated mVCP mice ${ }^{27}$. These findings were instrumental in advancing arimoclomol to a randomized, double-blinded, and placebo-controlled, proof-of-concept trial for the treatment of sporadic IBM, which concluded that arimoclomol was safe and well tolerated in patients, with exploratory efficacy data showing trends towards improvement in physical function and muscle strength in the arimoclomol treated group ${ }^{27}$. A phase $2 / 3$ efficacy study of arimoclomol for the treatment of sporadic IBM is currently ongoing (ClinicalTrials.gov Identifier: NCT02753530). Furthermore, a double-blind, placebo-controlled safety and tolerability trial of arimoclomol in patients with rapidly progressive SOD1 ALS (which also recorded preliminary efficacy data) showed that arimoclomol is safe and well-tolerated and although not powered for therapeutic effect, the results of the efficacy outcome measures suggested a possible therapeutic benefit of arimoclomol ${ }^{28}$. Additional clinical and preclinical studies have demonstrated that arimoclomol crosses the blood brain barrier and is well tolerated ${ }^{29-31}$. Based on these findings, a Phase 3, Randomised, Placebo-Controlled trial of arimoclomol in ALS is currently underway (ClinicalTrials.gov Identifier: NCT03491462).

Since mVCP mice have been reported to also display spinal cord and brain pathology which is similar to that observed in MSP, and which reflects ALS and FTD ${ }^{26}$, respectively, in this study we examined the potential beneficial effects of arimoclomol on the ALS pathology and FTD pathology that is present in these mice. We observed a significant improvement in the pathology present in both the spinal cord and brain of mVCP mice following treatment with arimoclomol. Furthermore, we also examined the effect of augmenting the HSR by arimoclomol treatment in human cellular models, including mutant VCP patient fibroblasts and iPSC-derived motor neurons, and found that arimoclomol rescues key degenerative features in these mVCP patient cells in vitro. Importantly, the pathological features that were improved by arimoclomol in mVCP mice and VCP patient cells were also found to be present in in post-mortem brain tissue from patients with FTD, confirming the relevance of the findings in our experimental models. These results provide further evidence that amplification of the HSR by treatment with arimoclomol may be a beneficial therapy for both non-SOD1 ALS as well as FTD.

\section{MATERIALS AND METHODS}

\section{Breeding and maintenance of mutant VCP mice}

All experimental work was carried out under licence from the UK Home Office (Scientific Procedures Act 1986) and was approved by the Animal Welfare and Ethical Review Board of UCL Institute of Neurology. Transgenic mice overexpressing the wild-type or mutant (A232E) human VCP gene under the cytomegalovirus (CMV)-enhanced chicken beta-actin (Custer et al. 2010) were generated at St Jude Children's Research Hospital, Memphis, TN, USA and a colony established and maintained at UCL, UK. These mice had been repeatedly backcrossed to C57-Black-6 mice. The colony was 
184 increased by breeding transgenic VCP female mice to C57-Black-6 wildtype males. Only male 185 offspring were used in this study to prevent gender differences. Offspring were genotyped by standard 186 PCR and gel electrophoresis protocols.

187 All mice used in this study were housed in a temperature and humidity-controlled environment 188 maintained on a 12-h light/dark cycle. Food and water were provided ad libitum. In these experiments, 189 wildtype VCP (wtVCP) mice were used as a transgenic control for the mutant VCP (mVCP) mice.

\section{Arimoclomol treatment regime}

192 Wildtype and mutant VCP mice were treated with either arimoclomol (obtained from Orphazyme A/S.) 193 or vehicle (water). Following genotyping, mice were randomly divided into the following treatment 194 groups: (i) Non-transgenic wildtype mice treated with water alone (WT) (ii) Transgenic wildtype mice 195 treated with water alone (wtVCP) (iii) A232E mutant VCP mice with water alone (mVCP) (iv) A232E 196 mutant mice treated with arimoclomol $(\mathrm{mVCP}+\mathrm{A})$ and $(\mathrm{v})$ Transgenic wildtype mice treated with arimoclomol. Mice were weighed fortnightly to adjust arimoclomol dosage at $120 \mathrm{mg} / \mathrm{kg}$. Arimoclomol was diluted in drinking water and mice were treated from 4 months of age (start of symptomatic stage) to time of examination at 14 months. All experiments were undertaken blinded to genotype and treatment.

\section{Assessment of motor unit number}

203 For in vivo electrophysiology experiments, mice were deeply anesthetized with $1.5-2.0 \%$ isoflurane in 204 oxygen delivered through a Fortec vaporizer (Vet Tech Solutions Ltd.). The distal tendon of the 205 extensor digitorum longus (EDL) muscles in both hindlimbs were exposed and dissected free of other 206 tendons before being attached by silk thread to isometric force transducers (Dynamometer UFI 207 Devices, Welwyn Garden City, UK). The sciatic nerve was exposed and sectioned, and all branches were cut except for the deep peroneal nerve that innervates the EDL muscles. Isometric contractions were elicited by stimulating the sciatic nerve using square-wave pulses of $0.02-\mathrm{ms}$ duration at supramaximal intensity, using silver wire electrodes. The number of motor units in the EDL muscles

211 was assessed by stimulating the motor nerve with stimuli of increasing intensity, resulting in stepwise 212 increments in twitch tension because of successive recruitment of motor axons. The resulting traces 213 were counted to obtain total number of motor units. Force transducers were connected to a PicoScope 2143423 oscilloscope (Pico Technology) and subsequently analysed using PicoScope software v5.16.2 215 (Pico Technology). All experiments were carried out at room temperature $\left(23^{\circ} \mathrm{C}\right) . \mathrm{n}=7$ animals for 216 the wtVCP + arimoclomol group. For all other experimental groups $n=10$ or greater.

\section{Histochemistry}

219 For motor neuron counts, following administration of terminal anaesthesia (pentobarbitone injection) 220 mice underwent transcardiac perfusion with $4 \%$ paraformaldehyde. The lumbar region of the spinal 
cord and the complete brain was removed and $20-\mu \mathrm{m}$ transverse sections were cut. For motoneuron

222 survival sections were stained with gallocyanin (a Nissl stain). Motoneurons located within the sciatic 223 motor pool, in which the nucleolus was visible, were counted in each ventral horn on every third section 224 between L2 and L5 levels of the spinal cord. $n=5$ animals per experimental group.

For immunofluorescent labelling, frozen sections were blocked for 1 hour at room temperature in blocking solution (10\% normal goat serum in PBS $+0.1 \%$ Triton $X-100)$, followed by incubation with primary antibodies against the C-terminal of TDP-43 (ProteinTech 12892-1-AP Rabbit polyclonal 1:400), ubiquitin (GeneTex GT7811 Mouse monoclonal 1:500), HSP70 (Santa Cruz, W27 Mouse monoclonal 1:100), GFAP-Cy3 (Sigma G-A-5 Mouse monoclonal 1:1000), $\beta$-III tubulin (Cambridge 231 Bioscience 3525-100 Rabbit polyclonal 1:100 or Thermofisher 236-10501 mouse monoclonal 1:100), 232 Tia1 (Abcam ab205063 Rabbit polyclonal 1:50), p62 (Abcam ab56416 Mouse monoclonal 1:200), LC3 233 (Novus biologicals NB100 Rabbit polyclonal 1:500), for 1 hour at room temperature. Sections were washed in PBS and incubated for 2 hours at room temperature with the appropriate fluorescently labelled secondary antibodies. 4', 6-Diamidino-2-Phenylindole (DAPI; Sigma) incubation to label nuclei or Fluoromyelin Red myelin stain (Thermo Fisher F34652 1:300) to label myelin was performed for up to 1 hour. Sudan black was applied to sections for 10 minutes to quench autofluorescence prior to coverslip mounting. Brain and spinal cord sections from three mice per experimental groups were assessed for each antibody tested and compared to negative controls run simultaneously.

Fluorescent images were visualised under a Leica fluorescent microscope and analysed using Leica Application Suite software (Leica Microsystems, Germany).

\section{Assessment of motor neuron soma area}

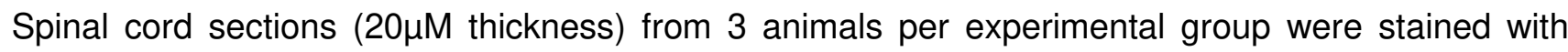
gallocyanine. 10 images of spinal cord regions L4 and L5 were taken at x20 magnification. Motor neurons from sciatic pool of left and right ventral horn in each section were drawn around to assess the total area of each cell using the Leica Application Suite software (Leica Microsystems, Germany).

\section{HSP70 intensity measurement}

251 HSP70-labelled immunofluorescent images of spinal cord sections from 3 animals per experimental 252 group were taken using a Zeiss LSM 780 confocal microscope under the same microscope and 253 imaging settings, at $x 40$ magnification. Motor neurons were identified by expression of $\beta$-III tubulin as 254 described above (labelled with Alexa fluor 568, red secondary antibody). Using Image $\mathrm{J}$ software each 255 neuron was drawn around and the total cell fluorescent intensity (for HSP70 labelled with Alexa fluor 256 488, green secondary antibody) per cell was measured, correcting for background fluorescence and 
cell area. An average of 50 cells per group were analysed from at least 5 different fields of view and the corrected mean intensities generated.

\section{Western blots}

261 Tissue samples and patient fibroblast cultures were homogenised in RIPA buffer (2\% SDS, $2 \mathrm{mM}$ EDTA, 2 mM EGTA in 5mM Tris, pH 6.8) and spun at 14,000 rpm for 15 minutes to pellet the cells.

Protein concentration was determined using a colorimetric protein assay system according to manufacturer's instructions (Bio-Rad Laboratories). Plates were incubated for 15 minutes at room temperature before absorbance was measured at $750 \mathrm{~nm}$ on a spectrophotometer. Homogenised samples in Laemmli sample buffer were loaded on acrylamide gels run at $160 \mathrm{~V}$ for 1 hour. Proteins were transferred onto a nitrocellulose membrane (Amersham) by running at $90 \mathrm{~V}$ for 70 minutes.

Blots were blocked in TBS+ $0.1 \%$ Tween $20+5 \%$ bovine serum albumin for 1 hour at room temperature before incubating overnight at $4^{\circ} \mathrm{C}$ with primary antibody anti-HSP70 (Santa Cru W27, Mouse monoclonal) at 1:1,000 dilution. Actin (Abcam ab8226 mouse monoclonal) or $\alpha$-tubulin (Sigma Aldrich DM1A Mouse monoclonal) were used as loading controls. Membranes were washed in either PBS+ $0.1 \%$ TWEEN or TBS+ $0.1 \%$ TWEEN and then incubated in HRP-conjugated secondary antibodies (Dako, 1:1000; Thermo Scientific, 1:500) for 2 hours at room temperature. StrepTactin (BioRad; $1: 10,000)$ was also added for visualisation of the protein ladder. Blots were visualised using Supersignal chemiluminescent HRP substrate (Thermo Scientific). Densitometry was analysed using ImageJ software (National Institutes of Health, Bethesda, MD, USA). Densities for the samples were normalized against densities of the loading control in each blot.

Four VCP patient fibroblast lines and three age-matched healthy control lines were obtained from Professor Hanns Lochmüller at the MRC Centre Neuromuscular Biobank (Newcastle University). Patient samples were from 3 individuals with the VCP R155H mutation (mild pheonotype) and one patient with R93C mutation (severe phenotype). Collection of samples from patients and healthy individuals and their use in research have been ethically approved by the 'Newcastle and North Tyneside 1 Research Ethics Committee' with REC reference number 08/HO906/28 + 5 with signed written consent obtained from patients.

Fibroblasts were grown in tissue culture flasks or 24 well plates in fibroblast media (10\% fetal bovine serum and 2\% PenStrep in Dulbecco's Modified Eagle Medium (DMEM) GlutaMAX-I), which was changed every 2-3 days. Cells were sub-cultured upon reaching $90 \%$ confluence using standard sub- 
294 culture and storage techniques. All cells used for experiments were used at passage 2-6 and 295 maintained at $37^{\circ} \mathrm{C}$ with $5 \% \mathrm{CO}_{2}$.

\section{Arimoclomol treatment of fibroblast cultures}

298 When cells reached $\sim 60 \%$ confluency in 24-well plates, cultures were treated with $10-400 \mu \mathrm{M}$ arimoclomol directly into media for 24 hours. Control cells were left untreated.

\section{Fibroblast Immunocytochemistry}

Fibroblasts were cultured on glass coverslips in 24-well plates and upon reaching approximately $70 \%$ confluency, fixed with 4\% paraformaldehyde (PFA). Following fixation, cells were incubated for 1 hour at room temperature with $10 \%$ normal goat/donkey serum in $0.1 \%$ PBS-Triton-X100 followed by overnight incubation at $4^{\circ} \mathrm{C}$ with primary antibodies: TDP-43 (ProteinTech 12892-1-AP Rabbit polyclonal 1:500), ubiquitin (GeneTex GT7811 Mouse monoclonal 1:500), p62 (Abcam ab56416 Mouse monoclonal 1:100). Phalloidin Alexa Fluor ${ }^{\text {TM }} 488$ (Thermo Fisher; $1: 200$ ) was used to visualise actin cytoskeleton of fibroblasts. One-hour incubation with fluorescently labelled secondary antibodies goat/donkey anti-rabbit/ mouse Alexa568 or goat/donkey anti-rabbit/mouse Alexa488 (1:500; Invitrogen) followed. Nuclear marker DAPI (1:2000) was added for 15 mins before coverslips were mounted onto glass slides. Negative controls omitting primary antibodies were carried out in parallel for all experiments.

314 Fluorescent images were visualised under a Leica DMR microscope and analysed using Leica Application Suite software (Leica Microsystems, Germany).

\section{TDP-43 nuclear intensity analysis}

318 To determine the nuclear intensity of TDP-43, fibroblasts were stained for TDP-43 and DAPI to outline the nuclear region of fibroblasts. A series of five images were taken at $20 x$ magnification, from one coverslip for each control or patient cell line, using a Leica DMR microscope. The corrected total cell fluorescence (CTCF) was determined using ImageJ and calculated as follows: CTCF= Integrated density - (area x mean fluorescence of background readings). Approximately 400-500 nuclei were analysed in each of the control and patient cell lines.

\section{Disrupted nuclei count}

326 To assess the percentage of cells with morphological nuclear abnormalities, fibroblasts were stained with DAPI to outline the nuclear region of fibroblasts. A series of ten images were taken at $20 \mathrm{x}$ magnification, from one coverslip for each control or patient cell line, using a Leica DMR microscope.

329 The number of cells with nuclear abnormalities were manually counted using ImageJ and the 330 percentage of the total number of cell nuclei calculated. Counts in approximately $400-800$ cells were 
331 undertaken in each of the control and patient cell lines. This was repeated in three separate

332 experiments. All experiments were undertaken blind to experimental group.

\section{4 iPSC culture and motor neuron differentiation}

335 iPSC cultures represent four clonal lines from 3 VCP patients and four healthy controls. iPSCs were 336 maintained on Geltrex (Life Technologies) with Essential 8 Medium media (Life Technologies), and 337 passaged using EDTA (Life Technologies, $0.5 \mathrm{mM}$ ). All cell cultures were maintained at $37^{\circ} \mathrm{C}$ and $5 \%$ 338 carbon dioxide. Motor neuron (MN) differentiation was carried out using a previously published 339 protocol (Hall et al., 2017). Briefly, iPSCs were first differentiated to neuroepithelium by plating to $340100 \%$ confluency in chemically defined medium consisting of DMEM/F12 Glutamax, Neurobasal, 341 LGlutamine, N2 supplement, non-essential amino acids, B27 supplement, $\beta$-mercaptoethanol (all from 342 Life Technologies) and insulin (Sigma). Treatment with small molecules from day 0-7 was as follows: $3431 \mu \mathrm{M}$ Dorsomorphin (Sigma), $2 \mu \mathrm{M}$ SB431542 (Sigma), and $3 \mu \mathrm{M}$ CHIR99021 (Sigma). At day 8, the 344 neuroepithelial layer was enzymatically dissociated using dispase (GIBCO, $1 \mathrm{mg} / \mathrm{ml}$ ), plated onto 345 Geltrex coated plates and next patterned for 7 days with $0.5 \mu \mathrm{M}$ retinoic acid and $1 \mu \mathrm{M}$ 346 Purmorphamine. At day 14 spinal cord MN precursors were treated with $0.1 \mu \mathrm{M}$ Purmorphamine for 347 a further 4 days before being terminally differentiated in $0.1 \mu \mathrm{M}$ Compound $\mathrm{E}$ (Sigma) to promote cell 348 cycle exit. Cells were treated with $50 \mu \mathrm{M}$ arimoclomol 5 days after terminal differentiation and fixed 349 with 4\% PFA after a further 24 hours.

351 The immunostaining protocols used for iPSC derived motor neurons were the same as that for 352 fibroblasts. Additional antibodies used for iPSCs were HSP70 (Santa Cruz Mouse monoclonal 1:100) 353 and $\beta$-III tubulin (TUJ1, Cambridge Bioscience 3526-100 Rabbit polyclonal 1:100).

\section{Post-mortem FTD tissue}

356 Post-mortem brain tissue was obtained from the Queen Square Brain Bank for Neurological disorders, 357 UCL Institute of Neurology, Wakefield Street, London WC1N 1PJ. Frozen cortical sections and frozen 358 tissue blocks were obtained from four patients with FTD subtypes and three healthy controls. See 359 Supplementary Table 1 for further information.

\section{Statistical analysis}

362 All data are presented as mean \pm SEM. Analyses were performed using GraphPad Prism analysis 363 software to determine the presence of statistically significant differences $(p<0.05)$ using unpaired t364 tests or two-way analysis of variance (ANOVA) as appropriate. For motor neuron counts, 365 experimental groups were compared using ANOVA test with Tukey's post hoc analysis. The 366 investigator was blind to experimental conditions of each animal where possible and appropriate. 
367 For disrupted nuclei counts, experimental groups were compared using a two-way analysis of variance 368 (ANOVA) with Tukey's all pairwise multiple comparisons post hoc analysis.

369 For TDP-43 nuclei intensity measurements, experimental groups were compared using a one-way 370 analysis of variance (ANOVA) with Tukey's all pairwise multiple comparisons post hoc analysis.

\section{RESULTS}

\section{Arimoclomol improves the ALS-like phenotype of mVCP mice.}

375 We have previously reported that mice expressing the human transgene for mutant VCP (A232E) have significant muscle pathology and reduced muscle strength ${ }^{27}$. As shown in Figure 1, electrophysiological analysis of mVCP mice at 14 months of age revealed that these mice also manifest significant motor deficits, reminiscent of ALS ${ }^{20}$. Thus, compared to control mice expressing wild-type human VCP (wtVCP), there was a $26.5 \%$ ( $p=0.019$, 1 -way ANOVA) reduction in the number of motor units innervating the hindlimb extensor digitorum longus (EDL) muscle in mVCP mice (Fig. $1 \mathrm{~A}$ and $1 \mathrm{~B}$ ), decreasing from an average of $34 \pm 1.5$ motor units in wtVCP mice to $25 \pm 2$ in mVCP mice. The loss of functional motor units is the defining characteristic of ALS which accounts for the loss of innervation at the neuromuscular junction and the resulting muscle paralysis.

Treatment of $\mathrm{mVCP}$ mice with $120 \mathrm{mg} / \mathrm{kg}$ of arimoclomol daily in drinking water, from 4 months (symptom onset) to 14 months of age resulted in a complete prevention of motor unit loss in EDL muscles. In the arimoclomol-treated mVCP cohort, $35 \pm 1.9$ motor units innervated the EDL muscle $(p=0.0017)$, which is similar to the number in both wtVCP mice $(34 \pm 1.5)$ and WT non-transgenic littermate controls $(34.3 \pm 0.9$; set as $100 \%$, Fig. 1B). There was no significant difference in the number of EDL motor units between the two control groups wtVCP and non-transgenic WT littermates (2-way ANOVA).

Following these acute physiological experiments, the spinal cord and brain were removed for histopathological analysis. Quantification of the number of motor neurons in the sciatic motor pool which innervate hind limb muscles, showed no significant difference in the number of motor neurons in wtVCP and non-transgenic WT controls, in which there were $725 \pm 9$ and $692 \pm 12$ motor neurons, respectively. However, there was a significant drop in motor neuron survival in mVCP mice, in which only $494 \pm 6$ motor neurons survived; this represents a $32 \%$ reduction in motor neuron survival compared to wtVCP controls ( $p=0.0001$, 2-way ANOVA; Fig. $1 C$ and D). 
401 Treatment with arimoclomol significantly improved motor neuron survival in mVCP mice, and $612 \pm$ 40218 motor neurons survived representing an improvement of $17 \%(p=0.029)$ when compared to 403 untreated mVCP mice. Importantly, arimoclomol had no effect on motor neuron survival in wtVCP 404 mice, so that $708 \pm 24$ motor neurons survived compared to $725 \pm 9$ in untreated wtVCP mice.

405 Furthermore, in untreated mVCP mice, motor neurons which survived at 14 months, had an abnormal 406 morphology, with small, compacted cell bodies, contrasting with the large polygonal shape of motor 407 neurons observed in control animals (Fig. 1C). We therefore assessed the total soma area of motor 408 neurons in the sciatic pool in each cohort of mice. As can be seen in Fig. 1E, there was a clear 409 reduction in the mean soma size of the motor neurons that survived in mVCP mice compared to 410 wtVCP controls. Size distribution analysis revealed that the reduction in mean motor neuron soma 411 area in $\mathrm{mVCP}$ mice was predominantly due to the preferential loss of large, likely alpha motor neurons, 412 and an increase in the proportion of smaller motor neurons (Fig. 1F). This shift in the size distribution 413 of motor neuron soma size in mVCP mice was prevented in arimoclomol-treated mVCP mice, in which 414 the motor neuron soma size distribution was similar to that observed in control mice (Fig. 1F).

416 Aggregation of misfolded proteins in ubiquitin containing inclusions and cytoplasmic mislocalisation of 417 the nuclear RNA-binding protein TDP-43 are key hallmarks of ALS pathology. We observed no 418 ubiquitin pathology in the spinal cord of wtVCP transgenic or non-transgenic WT littermates. In 419 contrast, ubiquitin-positive protein aggregates were observed in motor neurons of mVCP mice (Fig. 420 2A). While immunostaining for cytoplasmic TDP-43 was almost undetectable in wtVCP tissue, a 421 distinct increase in cytoplasmic TDP-43 was observed in motor neurons of mVCP mice (Fig. 2B). In 422 contrast, in mVCP mice treated with arimoclomol, cytoplasmic TDP-43 mislocalisation and ubiquitin 423 pathology was rarely observed, with immunostaining for ubiquitin and cytoplasmic TDP-43 similar to 424 that seen in control animals (Fig. 2B).

HSP70 levels from individual neurons in the spinal cord were quantified to better understand the HSR response in these cells. The pathological changes observed in the spinal cord of mVCP mice were associated with a 2-fold increase in the expression of HSP70 in motor neurons, compared to that in 429 wtVCP control animals in which there was no pathology (Fig. 2C and 2D). Importantly, this indicates 430 that the endogenous response to cell stress can and has been triggered in the mVCP motor neurons, 431 although this was not sufficient to prevent the development of pathology. In mVCP mice treated with 432 arimoclomol, an amplifier of the HSR, an even greater increase in HSP70 expression was observed, 433 to approximately 3-fold that of controls (Fig. 2C and 2D). In addition, in arimoclomol-treated mVCP 434 mice, GFAP-positive astroglia were also found to express increased levels of HSP70 in the spinal 435 cord (Fig. 2E), which likely reflects an additional cytoprotective response to mVCP-induced stress. 
VCP is known to play an essential role in autophagy ${ }^{10}$, and dysfunctional autophagy has been 438 implicated in the pathogenesis of ALS and MSP. For example, MSP patients expressing mVCP show 439 evidence of disrupted autophagy, with accumulation of the key autophagic markers p62 440 (Sequestosome 1) and LC3 within myofibres ${ }^{10,32}$. We therefore examined the pattern of expression 441 of these two autophagic markers in the spinal cord of mVCP mice. As can be seen in Figure 3, we 442 observed an increase in p62 expression in both the white and grey matter of the spinal cord compared 443 to control animals (Fig. 3A and B), with p62 aggregates clearly visible in motor neurons (Fig. 3B i444 magnified inset). Co-labelling with fluoromyelin suggested that the intense p62 staining observed in mVCP spinal cord sections was associated with oligodendrocytes (Fig. 3A ii). Closer examination of the p62-positive oligodendrocytes revealed gross myelin disruption around axons, deviating from the classic 'onion bulb' structure of healthy myelin, suggestive of axonal and/or myelin degeneration (Fig. 3B ii). This pattern of p62 expression was not observed in spinal cords from any control animals and was largely prevented in arimoclomol treated animals.

We also examined the expression pattern of the autophagosome marker LC3. LC3 is recruited to the autophagosomal membrane during autophagy and later degraded in the autolysosomal lumen, and as such is routinely used as a marker of autophagic activity in cells ${ }^{33,34}$. In mVCP mice we observed a significant increase in the expression of LC3 in the spinal cord and in particular in oligodendrocytes associated with abnormal myelination. Together with the accumulation of $p 62$, this provides further evidence of defective autophagy in these cells due to the presence of mutant VCP (Fig. 3C). Importantly, the accumulation of p62 and LC3 and abnormal myelination was significantly ameliorated in arimoclomol-treated mVCP mice (Fig. $3 \mathrm{~A}$ iii and $3 \mathrm{C}$ iii).

\section{Arimoclomol improves the FTD-like phenotype in the brain of mVCP mice.}

461 A third of patients diagnosed with MSP caused by mutations in VCP develop FTD ${ }^{7}$ and mutations in VCP cause $<1 \%$ of all FTD cases ${ }^{35}$. To determine the extent that this may be replicated in our mouse model, we next examined the brain of mVCP mice for FTD-like pathology.

Similar to ALS and MSP, cytoplasmic mislocalisation of TDP-43 is a pathological characteristic of FTD, with mislocalised TDP-43 present in the brain of approximately $50 \%$ of FTD cases, and brain pathology often indistinguishable from that seen in patients with a mutation in the TARDBP gene itself 7, 36. Cytoplasmic TDP-43 is often found either dispersed in the cytosol or within inclusion bodies, concomitant with its nuclear clearance ${ }^{37}$, indicating that a loss of normal nuclear function as well as gain of toxic cytoplasmic function may play a role in disease pathogenesis ${ }^{36}$. In this study we observed an increase in the number of cortical neurons with distinct cytoplasmic TDP-43 mislocalisation in mVCP mice compared to controls, with many neurons showing nuclear clearance of TDP-43 (Fig. 4A). In contrast, TDP-43 expression in the cortex of arimoclomol-treated mVCP mice was similar to control 
animals, as observed in the spinal cord, and no cytoplasmic mislocalisation associated with nuclear clearance of TDP-43 was apparent in the brains of the arimoclomol-treated group ( $n=3$ mice, Fig. 4A).

We next examined the expression of ubiquitin in the brain of mVCP mice. Similar to our findings in the spinal cord, in mVCP mice we observed ubiquitin-positive intracellular aggregates in the brain, although these were not limited to the cortex. No ubiquitin-positive aggregates were detected in control animals, or in mVCP mice treated with arimoclomol (Fig. 4B).

Microtubule-associated protein tau (MAPT) is associated with a well-known genetic form of FTD and deposits of $p$-tau are often detected in post-mortem brain of dementia patients ${ }^{38}$. We found extracellular deposits of phosphorylated tau (p-tau, AT8) in the brain of mVCP mice, which were not observed in WT control mice (Supplementary Fig. 1 A), and which may have formed through nonspecific aggregation of proteins. The tau deposits were often observed surrounded by lba1-positive microglia or GFAP-positive astrocytes, suggesting an inflammatory response to the abnormal presence of $p$-tau (Supplementary Fig. $1 \mathrm{~A}$ ). In contrast, in control and arimoclomol-treated mVCP mice, no tau-positive deposits were observed in any area of the brain assessed.

While TDP-43 plays an important role in RNA metabolism, mislocalisation of this protein suggests that other RNA-associated proteins may also be affected in mVCP mice. Stress granule formation is a highly evolutionarily conserved cytoprotective mechanism to temporarily store stalled translation preinitiation complexes during episodes of cellular stress ${ }^{39}$. We examined the expression of Tia1, an RNA-binding protein, known to be present in stress granules ${ }^{40}$ in mouse brain tissue. We discovered that Tia1 co-localised with both TDP-43 and ubiquitin inclusions in the cytoplasm of mVCP brains suggesting the possible formation of non-specific protein aggregation (Fig. 4C). It has been demonstrated in vitro that TDP-43-containing granules in the cytoplasm may seed aggregation through RNA binding 41, 42. Furthermore, regenerating myofibres reportedly contain TDP-43 'myogranules' which may be the precursors for aggregation in diseased muscle, as shown in mutant VCP (A232E) mouse muscle ${ }^{41}$. It is therefore possible that a similar phenomenon also occurs in the brain of $\mathrm{mVCP}$ mice, leading to the aggregates observed in this study. Two additional markers of stress granules, FMRP and G3BP were also associated with protein aggregates in the brain of mVCP mice (Supplementary Fig. 1 B). In control animals and mVCP mice treated with arimoclomol, no aggregates positive for any of the tested stress granule markers were observed (Supplementary Fig. $1 \mathrm{~B}$ ).

Furthermore, as observed in the spinal cord, immunostaining of mVCP mouse cortex also revealed the presence of p62 and LC3-positive aggregates, with intense cytosolic LC3 staining (Fig. 5A and B). No LC3 staining was observed in control tissue or arimoclomol treated mVCP mice. 
511 Similar to the findings in the spinal cord of mVCP mice, brain tissue from these animals also showed 512 an increase in the expression of HSP70 compared to wtVCP and non-transgenic WT controls, 513 indicative of stress-induced activation of the HSR. In mVCP mice treated with arimoclomol, HSP70 514 expression was found to be further increased, suggesting that the HSR has been augmented by 515 arimoclomol treatment (Fig. 5C). Interestingly, as noted in the spinal cord, HSP70 expression was 516 significantly pronounced in glial cells in the brain, which are known to have a robust stress induced 517 HSR.

\section{Pathology observed in mVCP mice reflects that observed in ALS/FTD patient brain tissue.}

521 by treatment with arimoclomol, have relevance to the human disease, we examined post-mortem brain 522 tissue from 4 patients with FTD (diagnosed as FTD patient with: motor neuron disease (FTD-MND), 523 ubiquitin-positive inclusion bodies (FTD-U), mutation in TDP-43 (FTD-TDPA) or with mutant tau 524 protein (FTD-MAPT)). A panel of the same markers used in the mVCP mice were assessed and results compared to samples of the same region from age-matched healthy human brains.

Since autophagy is a common pathway that may be disrupted in FTD brain regardless of specific disease aetiology, we examined human FTD brain for the presence of autophagy-related proteins which were abnormally present in mVCP mice. Globular, juxtanuclear p62-positive cytoplasmic inclusion bodies were present in cortical brain sections of the FTD cases (Fig. 6A) and were similar to those seen in mVCP mice (Fig. 5A), suggesting a generalised disruption in protein homeostasis in FTD patients regardless of subtype; no p62 immunostaining was observed in brain sections from healthy controls. Strong p62 staining was also observed in the FTD-MAPT brain (Fig. 6A), similar to that reported in the brain of Alzheimer's disease patients early in pathogenesis within neurofibrillary tangles ${ }^{43}$. In FTD-TDPA brain, intense p62 staining was observed in neural processes in addition to cytoplasmic inclusion bodies. Interestingly, a similar pattern of TDP-43 staining has been reported in the upper cortical layers in FTD-TDPA patients ${ }^{44}$.

Furthermore, immunostaining of cortical sections revealed the presence of LC3-positive aggregates in all FTD patients, similar to findings in mVCP mice (Fig 5B). FTD-MAPT and FTD-TDPA samples also contained cells with more intense cytoplasmic staining throughout cells than seen in FTD-U and FTD-MND patients, which corresponded to significantly increased LC3 I levels detected by immunoblot (FTD-MAPT patient, $p=0.043$ and FTD-TDPA, $p=0.015$, 2-way ANOVA) compared to healthy controls (Supplementary Fig. 2). It is likely that total LC3 I level in samples with less cytoplasmic staining are also above basal levels but not detected in the soluble lysates due to aggregation. No significant difference in LC3 II, which closely correlates to the number of autophagosomes, was detected by immunoblot. 
548 Although TDP-43 mislocalisation in FTD brain is a well-established phenotype, we demonstrate 549 extensive cytoplasmic mislocalisation of TDP-43 in the brain of all four FTD patients in which p62 and 550 LC3 are also aggregated. Such mislocalisation was not observed in samples from healthy controls 551 (Fig. 6C). Our findings thus reveal that the pathological phenotypes identified in the mVCP mouse 552 model are indeed present in the human disease. Moreover, these phenotypes are not limited to 553 mutations in VCP, therefore expanding the relevance of our findings of the beneficial effects of 554 arimoclomol to non-VCP FTD patients.

556 While the ability to mount a HSR under conditions of cell stress is present throughout life, this 557 cytoprotective mechanism is thought to become less effective in later life, likely contributing to the 558 age-related increase in susceptibility to degenerative diseases ${ }^{45}$. In our study, compared to age559 matched control post-mortem samples, HSP70 expression was indeed significantly lower in the cortex 560 of 2 out of 4 of the FTD patients examined (Fig. 6D). However, a reduced HSR is not a prerequisite 561 for treatment potential and therapeutically augmenting this endogenous cytoprotective process with 562 arimoclomol may be a beneficial strategy in response to neurodegenerative diseases such as 563 ALS/FTD.

Patient-derived mutant VCP fibroblasts and iPSC motor neurons develop pathology which is improved by arimoclomol treatment.

Our results show for the first time, that upregulation of the HSR by treatment with arimoclomol significantly ameliorates the pathological deficits observed in the brain and spinal cord of mVCP mice. To further test whether the beneficial effects of arimoclomol may have therapeutic relevance for human ALS/FTD, we next examined the effects of arimoclomol in two human models of ALS/FTD, by establishing two patient-derived cellular in vitro models of mutant VCP: i) mutant VCP patient fibroblasts, a relatively simple cellular system with defined mutations and the cumulative cellular damage of the patients; this model enabled us to test the effects of mutant VCP on human cells and to establish the effects of arimoclomol on non-neuronal cells; ii) human induced pluripotent stem cell (iPSC)-derived motor neurons (iPSC-MNs) established from ALS patients expressing mutant VCP, which provide a more complex, neuronal and highly disease-specific cell culture model of

Fibroblasts derived from patients with the most prevalent MSP-associated VCP mutation, $\mathrm{R} 155 \mathrm{H}$, have been reported to display some evidence of a pathological phenotype when grown in culture ${ }^{47}$. In this study, we assessed the distribution of a number of ALS/FTD-associated proteins in fibroblasts expressing either the $\mathrm{R} 155 \mathrm{H} V C P$ mutation, obtained from three patients with a mild disease phenotype or the R93C VCP mutation, from a patient with a more severe disease phenotype. We 
mVCP fibroblasts from all patients. The majority of p62-positive aggregates were located adjacent 586 to the nucleus (Fig. 7A), whilst ubiquitin-positive aggregates were present either as large, distinct 587 aggregates (Fig. 7B i) or as smaller, diffuse aggregates (Fig. 7B ii) that were more evenly dispersed 588 throughout the cytoplasm. Although this dispersed expression of ubiquitin was also noted in a small number of age-matched control fibroblasts (data not shown), large aggregates of ubiquitin or p62 were not detected in control cells or in patient fibroblasts after treatment with arimoclomol $(10 \mu \mathrm{M}, 24$-hour treatment).

No TDP-43-positive cytoplasmic aggregates were observed in control fibroblasts (Fig. 7C i). However, abnormal TDP-43 expression patterns were noted in mVCP fibroblasts, with differences observed between patient lines, potentially reflecting phenotypic differences between mild and severe cases. In some mVCP fibroblasts, TDP-43 was retained in the nuclei of cells in which cytoplasmic aggregates were present (Fig. 7C ii). However, several cells from the more severely affected mVCP R93C line showed evidence of TDP-43 nuclear depletion, even in the absence of cytoplasmic aggregates (Fig. 7C iii). This pattern was not seen in the milder $\mathrm{R} 155 \mathrm{H}$ line, control cells or in R93C cells treated with $10 \mu \mathrm{M}$ arimoclomol. Indeed, quantification of nuclear TDP-43 levels shows that arimoclomol treatment prevents the loss of TDP-43 in the nuclei of R93C cells (Fig. 7D).

The pathological changes observed in mVCP fibroblasts were also associated with an increase in the expression of HSP70 (Fig. 7E), which was almost 2-fold higher than that observed in control cells, indicating the presence of cell stress. Furthermore, we also observed a significant increase in the number of nuclei with abnormal morphology, consisting of herniations and fragmentation of nuclei leading to the generation of micronuclei, in both the R155H and R93C mVCP cell lines (Fig. $7 \mathrm{~F} \mathrm{i-} \mathrm{ii).}$ Surprisingly, these cells were not undergoing apoptosis, as assessed by TUNEL staining for DNA double-strand breaks (Supplementary Fig. 3). Quantification of the number of aberrant nuclei in mVCP fibroblasts in the absence and presence of increasing concentrations of arimoclomol revealed a dosedependent reduction in the number of disrupted nuclei, with a statistically significant difference observed at $50 \mu \mathrm{M}$ of arimoclomol (Fig. $7 \mathrm{~F} \mathrm{iii).}$

In order to confirm our results in a more relevant cellular model of ALS, we also investigated the effects of arimoclomol on iPSC-derived motor neurons from patients with VCP mutations (3 individual patient lines). TDP-43 cytoplasmic mislocalisation has been previously reported in iPSCs from patients with mutated VCP ${ }^{46}$. As expected, we frequently observed distinct cytoplasmic TDP-43 in mVCP IPSCderived motor neuron cultures, with many cells also exhibiting nuclear loss of TDP-43 (Fig. 8A, magnified image of cell). Importantly, mislocalised TDP-43 was rarely seen in the mVCP cultures treated with $50 \mu \mathrm{M}$ arimoclomol and was absent from healthy control cells. 
621 Ubiquitin-positive and p62 immuno-reactive protein aggregates were also detected in mVCP iPSC622 motor neurons, either as many small bodies dispersed throughout the cell or as one large distinct 623 globular aggregate within the cytoplasm (Fig. 8B and D). Quantification of cells with ubiquitin-positive 624 aggregates revealed a $22 \%$ increase in mVCP neurons compared to controls, with a significant 625 reduction (down to 11\%) in arimoclomol treated cultures (Fig $8 \mathrm{C}$ ). No statistical significance was identified between control and arimoclomol treated mVCP groups. These findings corroborate our observations in mVCP mice, where abnormal ubiquitin and p62 accumulation, possibly linked to impairment of autophagy or proteasomal degradation, was present in both the spinal cord and brain. More importantly, these data clearly show that arimoclomol treatment leads to a reduction of cytoplasmic ubiquitin aggregates in a specific neurological cellular system with VCP mutation.

As seen in the mVCP mice, expression of HSP70 was increased in mVCP iPSC-derived motor neuron cultures under basal conditions, indicating that these cells are under stress and have activated the HSR. Treatment of mVCP iPSC-derived motor neurons with arimoclomol resulted in a clear increase in HSP70 expression above that observed in untreated mVCP iPSC-MNs (Fig. 8E), indicating an enhancement of the endogenous cytoprotective HSR, which is likely to account for the improvement in protein mishandling pathology observed untreated cells.

\section{DISCUSSION}

We have previously demonstrated the beneficial effects of augmenting the HSR with arimoclomol on the ALS phenotype of mutant SOD1 mice ${ }^{11,12,13}$ as well as the IBM-like muscle pathology that is present in mutant VCP mice ${ }^{27}$. In the present study, we expand these findings to examine the effects of arimoclomol on the spinal cord and brain pathology which is also part of the MSP phenotype of mVCP mice, and which we show to be similar to pathological characteristics of human ALS/FTD.

Our results show that the loss of functional motor units and corresponding degeneration of spinal motor neurons observed in mVCP mice was ameliorated by treatment with arimoclomol. In particular, large, most likely alpha neurons, which were preferentially lost in mVCP mice were rescued by arimoclomol. The preferential degeneration of alpha motor neurons is a well-established feature of ALS ${ }^{48,49}$ and has been previously observed in the SOD1 ${ }^{\mathrm{G} 93 \mathrm{~A}}$ mouse model of ALS ${ }^{20}$. Importantly, this finding shows for the first time, that arimoclomol is able to rescue the defining pathological hallmark of ALS not only in models of SOD1-ALS mice ${ }^{11,12,13}$, but also in a model of non-SOD1 ALS, suggesting presence of ubiquitin-positive aggregates and cytoplasmic mislocalisation of TDP-43. Furthermore, 
658 we observed accumulation of p62 and LC3 in neurons and myelinating oligodendrocytes, suggesting 659 that deficits in autophagy may lead to inefficient clearance of aberrant proteins in mVCP spinal cord 660 neurons. In addition, we present evidence of myelin disruption in the spinal cord white matter, which 661 may reflect axonal and/or myelin degeneration. The beneficial effects of arimoclomol in ameliorating 662 these pathological features of ALS are likely to be due to an increase in the HSR, as they were 663 accompanied by a significant increase in the expression of HSP70 in both neurons and glia. These 664 observations are consistent with previous publications demonstrating that amplification of HSP70 665 improves lysosomal function and myelination in the CNS ${ }^{29}$.

A similar pathological phenotype was observed in the brain of mVCP mice, including the presence of ubiquitinated inclusion bodies, TDP-43 mislocalisation and accumulation of p62 and LC3 in neurons; these pathological markers were again associated with an increase in the expression of HSP70. Areas of p-tau pathology were also present, with Iba1 and GFAP-positive cells surrounding p-tau lesions, suggesting the presence of an inflammatory response. Stress granule markers, co-localised with ubiquitin and TDP-43, were also found in mVCP mouse brains. This indicates that there may be impaired stress granule clearance, as previously reported when VCP function has been inhibited ${ }^{50}$. Arimoclomol treatment improved all of the pathological changes observed in mVCP mouse brains and was associated with a clear enhancement in HSP70 expression above that occurring in untreated mVCP mice in both neurons and glia. This is consistent with previous reports demonstrating that arimoclomol readily crosses the blood brain barrier and amplifies the expression of HSP70 within the CNS ${ }^{29,31}$. Upregulation of the HSR in glial cells may reflect a stress response in these cells or may be part of a neuroprotective mechanism to help defend neurons against $\mathrm{mVCP}$-induced stress through the expression of heat shock proteins ${ }^{51}$. Glial HSP70 is known to be protective to neurons and exogenous HSP70 has been shown to be beneficial in improving motor neuron survival in the SOD1 ${ }^{\text {G93A }}$ mouse model of ALS ${ }^{52-54}$.

We also examined two human cellular models of mutant VCP disease, which provide more clinically relevant platforms to test the effects of arimoclomol and also allow for more quantifiable analysis of some of the observations made in the mouse and FTD patient post-mortem tissues. In both fibroblasts and iPSC-derived motor neurons from mVCP patients, the key degenerative phenotypes observed in $\mathrm{mVCP}$ mice were recapitulated, including the formation of ubiquitinated protein aggregates and TDP43 pathology and these changes were associated with an increase in HSP70 expression as seen in mVCP mice. While disperse cytoplasmic TDP-43 staining was not easily detectable in the mVCP fibroblasts, reduced nuclear TDP-43 (without clear cytoplasmic mislocalisation) has previously been described in fibroblasts from ALS VCP patients, where truncated and phosphorylated TDP-43 was associated with the nuclear reduction, and imply altered TDP-43 metabolism in these cells ${ }^{55}$. 
694 Arimoclomol treatment demonstrated an improvement in TDP-43 pathology in both human models 695 examined in this study.

697 Furthermore, abnormal nuclear morphology was observed in mVCP fibroblasts, which has previously 698 been reported in IBM patient muscle ${ }^{56}$. This phenomenon may be the result of altered nuclear membrane integrity brought about by an imbalance of proteins that constitute the nuclear lamina when protein homeostasis is disrupted ${ }^{57}$. Importantly, arimoclomol treatment demonstrated a significant and dose-dependent reduction in this aberrant nuclear morphology.

Thus, arimoclomol treatment ameliorated key pathological features assessed in human cells in vitro, corroborating our in vivo data from mVCP mice and demonstrating that i) the HSR can indeed be therapeutically manipulated in vitro in patient cells associated with ALS/FTD mutations, and ii) pharmacological augmentation of the HSR is sufficient to rescue key degenerative changes in human mVCP cells in vitro.

In parallel to the experimental data, we also confirm that the same key pathological characteristics of ALS/FTD observed in the mouse and recapitulated in the human cell models of mVCP disease, and which were ameliorated by treatment with arimoclomol, are present in cortical brain samples of FTD patients. Our results show that TDP-43 pathology was present in all FTD patient samples examined. In addition, both p62 and LC3 were found to be accumulated in FTD brains across the subtypes examined, either as soluble proteins detectable by immunoblot or as aggregates revealed by histochemistry. These findings suggest that impaired clearance of neurotoxic proteins by autophagy may be a common factor in FTD, and not necessarily specific to a particular mutation or aetiology.

Although the scale, kinetics and required threshold of the HSR varies between cells and stressors, this vital mechanism routinely keeps cells free of damaged and surplus proteins ${ }^{58}$. As a highly adaptive system, the HSR is able to tailor the type of stress response that is most appropriate for the type of cell involved and for the specific type of stress they are under ${ }^{59}$, coordinating stress-induced transcription of a variety of chaperones and co-chaperones. While we found the HSR to be induced in all the mVCP models we examined, the magnitude of the endogenous HSR mounted was clearly not sufficient to prevent pathology. Furthermore, the HSR, as measured by HSP70 levels, remained unaltered or deficient in FTD patient tissue which share common pathogenic hallmarks with our disease models. It has been long established that attenuation of the HSR is associated with aging and this may be, in part, responsible for the accumulation of aberrant proteins and thus altered regulation of cell death which we see in chronic neurodegeneration ${ }^{45,60}$. All FTD post-mortem brain samples investigated in this study were from patients of relatively advanced age, ranging from 62-79 years old and were compared to age-matched controls and as such, a lower HSR is not unexpected. Our data 
therefore suggests that a diminished HSR is not necessarily a precondition for treatment with a HSR

732 amplifier, and pharmacologically correcting any deficiency or amplifying the endogenous HSR using 733 arimoclomol may be an effective approach to alleviate neurodegenerative pathology and therefore 734 delay disease progression in ALS/FTD patients.

Importantly, when considering arimoclomol's potential as a therapeutic agent, its ability to act as an amplifier and not an inducer of the HSR; thereby reducing any potential off-target effects, is critical. In all the disease models examined in this study, we demonstrate that amplification of the HSR with arimoclomol prevents the development of pathology characteristic of protein dyshomeostasis, including the mislocalisation of TDP-43, and mitigates formation of ubiquitin-rich inclusion bodies. Moreover, we show that these improvements in cell health are associated with significantly improved neuronal survival in vivo, which is vital for slowing disease progression in neurodegenerative conditions such as ALS and FTD.

\section{Conclusions}

746 The results of this study show that in both spinal cord and brain of transgenic mice and in human cells, expression of mutant VCP gives rise to a neurodegenerative pathology that is reminiscent of ALS/FTD and MSP, including protein aggregation and TDP-43 mislocalisation, resulting in cell death. Furthermore, we show that these pathological features are also present in human FTD brain, irrespective of genetic subtype.

Taken together with the results from our previous studies in mVCP muscle, SOD1 ${ }^{\text {G93A }}$ ALS mice ${ }^{20-22}$ as well the AR100 mouse model of SBMA, our findings suggest that several common pathological characteristics develop when protein mishandling occurs in a cell, regardless of the cause (in both genetic and sporadic disease), the tissue type or indeed whether murine or human. Moreover, our results demonstrate the effectiveness of arimoclomol in ameliorating these pathological characteristics through an upregulation of cytoprotective chaperone proteins which are endogenous to all cell types.

Arimoclomol is a small molecule HSP amplifier that is clinically well-tolerated and readily crosses the blood-brain barrier. The results of this study thus suggest that arimoclomol treatment may be a disease-modifying therapeutic approach for the treatment of non-SOD1 ALS as well as MSP. Furthermore, since the pathology induced in mVCP mice and human cells in vitro mirrors that observed in human FTD patient post-mortem brain, our results also indicate the potential therapeutic value of arimoclomol for the treatment of FTD and potentially other neurodegenerative conditions with evidence of disrupted protein homeostasis. 
We would like to thank The Queen Square Brain Bank for providing the post-mortem brain samples used in this study. The Queen Square Brain Bank is supported by the Reta Lila Weston Institute of Neurological Studies, UCL Queen Square Institute of Neurology. We are grateful to the Rosetrees Trust for their support. LG is the Graham Watts Senior Research Fellow, supported by Brain Research 773 UK.

\section{Competing interests}

The authors report no competing interests.

\section{Author contributions}

MA undertook the in vivo study, the study on patient samples and contributed towards the iPSC data. CS undertook the in vitro study in human fibroblasts. JH led the iPSC study. JPT provided the transgenic mice breeders for the in vivo study. NHTP and TK are industry collaborators. MH and RP collaborating principal investigators and $L G$ is the lead principal investigator and corresponding author.

\section{RERERENCES}

1. Van Langenhove T, van der Zee J, Van Broeckhoven C. The molecular basis of the frontotemporal lobar degeneration-amyotrophic lateral sclerosis spectrum. Ann Med 2012;44:817828.

792 2. Majounie E, Renton AE, Mok K, et al. Frequency of the C9orf72 hexanucleotide repeat expansion in patients with amyotrophic lateral sclerosis and frontotemporal dementia: a crosssectional study. Lancet Neurol 2012;11:323-330.

795 3. Van Mossevelde S, Engelborghs S, van der Zee J, Van Broeckhoven C. Genotype-phenotype 796 links in frontotemporal lobar degeneration. Nat Rev Neurol 2018;14:363-378.

797 4. Dols-Icardo O, Garcia-Redondo A, Rojas-Garcia R, et al. Analysis of known amyotrophic 798 lateral sclerosis and frontotemporal dementia genes reveals a substantial genetic burden in patients 799 manifesting both diseases not carrying the C9orf72 expansion mutation. J Neurol Neurosurg 800 Psychiatry 2017.

801 5. Johnson JO, Mandrioli J, Benatar M, et al. Exome sequencing reveals VCP mutations as a 802 cause of familial ALS. Neuron 2010;68:857-864.

803 6. Ranganathan R, Haque S, Coley K, Shepheard S, Cooper-Knock J, Kirby J. Multifaceted 804 Genes in Amyotrophic Lateral Sclerosis-Frontotemporal Dementia. Front Neurosci 2020;14:684. 
805 7. Ng AS, Rademakers R, Miller BL. Frontotemporal dementia: a bridge between dementia and 806 neuromuscular disease. Ann N Y Acad Sci 2015;1338:71-93.

807 8. Li H, Chen Q, Liu F, et al. Unfolded protein response and activated degradative pathways 808 regulation in GNE myopathy. PloS one 2013;8:e58116.

809 9. Buchberger A, Schindelin H, Hanzelmann P. Control of $p 97$ function by cofactor binding. FEBS 810 Lett 2015;589:2578-2589.

811 10. Ju JS, Fuentealba RA, Miller SE, et al. Valosin-containing protein (VCP) is required for 812 autophagy and is disrupted in VCP disease. J Cell Biol 2009;187:875-888.

813 11. Schutz AK, Rennella E, Kay LE. Exploiting conformational plasticity in the AAA+ protein 814 VCP/p97 to modify function. Proc Natl Acad Sci U S A 2017.

815 12. Yeo BK, Hong CJ, Chung KM, et al. Valosin-containing protein is a key mediator between 816 autophagic cell death and apoptosis in adult hippocampal neural stem cells following insulin 817 withdrawal. Mol Brain 2016;9:31.

818 13. Morimoto RI. The heat shock response: systems biology of proteotoxic stress in aging and 819 disease. Cold Spring Harb Symp Quant Biol 2011;76:91-99.

820 14. Kaushik S, Cuervo AM. The coming of age of chaperone-mediated autophagy. Nat Rev Mol 821 Cell Biol 2018;19:365-381.

822 15. Barna J, Csermely P, Vellai T. Roles of heat shock factor 1 beyond the heat shock response. 823 Cell Mol Life Sci 2018;75:2897-2916.

824 16. Watanabe Y, Tsujimura A, Taguchi K, Tanaka M. HSF1 stress response pathway regulates 825 autophagy receptor SQSTM1/p62-associated proteostasis. Autophagy 2017;13:133-148.

826 17. Kirkegaard T, Roth AG, Petersen NH, et al. Hsp70 stabilizes lysosomes and reverts Niemann827 Pick disease-associated lysosomal pathology. Nature 2010;463:549-553.

828 18. Ingemann L, Kirkegaard T. Lysosomal storage diseases and the heat shock response: 829 convergences and therapeutic opportunities. J Lipid Res 2014;55:2198-2210.

830 19. Kaushik S, Cuervo AM. Chaperone-mediated autophagy: a unique way to enter the lysosome 831 world. Trends Cell Biol 2012;22:407-417.

832 20. Kieran D, Kalmar B, Dick JR, Riddoch-Contreras J, Burnstock G, Greensmith L. Treatment 833 with arimoclomol, a coinducer of heat shock proteins, delays disease progression in ALS mice. Nat 834 Med 2004;10:402-405.

835 21. Kalmar B, Edet-Amana E, Greensmith L. Treatment with a coinducer of the heat shock 836 response delays muscle denervation in the SOD1-G93A mouse model of amyotrophic lateral 837 sclerosis. Amyotroph Lateral Scler 2012;13:378-392.

838 22. Kalmar B, Novoselov S, Gray A, Cheetham ME, Margulis B, Greensmith L. Late stage 839 treatment with arimoclomol delays disease progression and prevents protein aggregation in the SOD1 840 mouse model of ALS. J Neurochem 2008;107:339-350. 
841 23. Malik B, Nirmalananthan N, Gray AL, La Spada AR, Hanna MG, Greensmith L. Co-induction 842 of the heat shock response ameliorates disease progression in a mouse model of human spinal and 843 bulbar muscular atrophy: implications for therapy. Brain 2013;136:926-943.

844 24. Hargitai J, Lewis H, Boros I, et al. Bimoclomol, a heat shock protein co-inducer, acts by the 845 prolonged activation of heat shock factor-1. Biochem Biophys Res Commun 2003;307:689-695.

846 25. Fog CK, Zago P, Malini E, et al. The heat shock protein amplifier arimoclomol improves 847 refolding, maturation and lysosomal activity of glucocerebrosidase. EBioMedicine 2018;38:142-153.

848 26. Custer SK, Neumann M, Lu H, Wright AC, Taylor JP. Transgenic mice expressing mutant 849 forms VCP/p97 recapitulate the full spectrum of IBMPFD including degeneration in muscle, brain and 850 bone. Hum Mol Genet 2010;19:1741-1755.

851 27. Ahmed M, Machado PM, Miller A, et al. Targeting protein homeostasis in sporadic inclusion 852 body myositis. Sci Transl Med 2016;8:331ra341.

853 28. Benatar M, Wuu J, Andersen PM, et al. Randomized, double-blind, placebo-controlled trial of 854 arimoclomol in rapidly progressive SOD1 ALS. Neurology 2018;90:e565-e574.

855 29. Kirkegaard T, Gray J, Priestman DA, et al. Heat shock protein-based therapy as a potential 856 candidate for treating the sphingolipidoses. Sci Transl Med 2016;8:355ra118.

857 30. Lanka V, Wieland S, Barber J, Cudkowicz M. Arimoclomol: a potential therapy under 858 development for ALS. Expert Opin Investig Drugs 2009;18:1907-1918.

859 31. Cudkowicz ME, Shefner JM, Simpson E, et al. Arimoclomol at dosages up to $300 \mathrm{mg} / \mathrm{day}$ is 860 well tolerated and safe in amyotrophic lateral sclerosis. Muscle Nerve 2008;38:837-844.

861 32. Weihl CC, Pestronk A, Kimonis VE. Valosin-containing protein disease: inclusion body 862 myopathy with Paget's disease of the bone and fronto-temporal dementia. Neuromuscul Disord 863 2009;19:308-315.

864 33. Tanida I, Ueno T, Kominami E. LC3 and Autophagy. Methods Mol Biol 2008;445:77-88.

865 34. Klionsky DJ, Abdelmohsen K, Abe A, et al. Guidelines for the use and interpretation of assays 866 for monitoring autophagy (3rd edition). Autophagy 2016;12:1-222.

867 35. Hortobagyi T, Cairns NJ. Amyotrophic lateral sclerosis and non-tau frontotemporal lobar 868 degeneration. Handb Clin Neurol 2017;145:369-381.

869 36. Ling SC, Polymenidou M, Cleveland DW. Converging mechanisms in ALS and FTD: disrupted 870 RNA and protein homeostasis. Neuron 2013;79:416-438.

871 37. Hiniker A, Daniels BH, Lee HS, Margeta M. Comparative utility of LC3, p62 and TDP-43 872 immunohistochemistry in differentiation of inclusion body myositis from polymyositis and related 873 inflammatory myopathies. Acta neuropathologica communications 2013;1:29.

874 38. Rademakers $R$, Cruts $M$, van Broeckhoven C. The role of tau (MAPT) in frontotemporal 875 dementia and related tauopathies. Hum Mutat 2004;24:277-295.

876 39. Monahan Z, Shewmaker F, Pandey UB. Stress granules at the intersection of autophagy and 877 ALS. Brain Res 2016;1649:189-200. 
878 40. Buchan JR, Parker R. Eukaryotic stress granules: the ins and outs of translation. Mol Cell 879 2009;36:932-941.

880 41. Vogler TO, Wheeler JR, Nguyen ED, et al. TDP-43 and RNA form amyloid-like myo-granules 881 in regenerating muscle. Nature 2018;563:508-513.

882 42. Nonaka T, Hasegawa M. [Intracellular seeded aggregation of TDP-43]. Rinsho Shinkeigaku 883 2012;52:1056-1058.

884 43. Kuusisto E, Salminen A, Alafuzoff I. Early accumulation of p62 in neurofibrillary tangles in 885 Alzheimer's disease: possible role in tangle formation. Neuropathol Appl Neurobiol 2002;28:228-237. 886 44. Tan $\mathrm{RH}$, Shepherd CE, Kril JJ, et al. Classification of FTLD-TDP cases into pathological 887 subtypes using antibodies against phosphorylated and non-phosphorylated TDP43. Acta Neuropathol 888 Commun 2013;1:33.

889 45. Verbeke P, Fonager J, Clark BF, Rattan SI. Heat shock response and ageing: mechanisms 890 and applications. Cell Biol Int 2001;25:845-857.

891 46. Hall CE, Yao Z, Choi M, et al. Progressive Motor Neuron Pathology and the Role of Astrocytes 892 in a Human Stem Cell Model of VCP-Related ALS. Cell Rep 2017;19:1739-1749.

893 47. Nalbandian A, Llewellyn KJ, Gomez A, et al. In vitro studies in VCP-associated multisystem 894 proteinopathy suggest altered mitochondrial bioenergetics. Mitochondrion 2015;22:1-8.

895 48. Nijssen J, Comley LH, Hedlund E. Motor neuron vulnerability and resistance in amyotrophic 896 lateral sclerosis. Acta Neuropathol 2017;133:863-885.

897 49. Kang SH, Li Y, Fukaya M, et al. Degeneration and impaired regeneration of gray matter 898 oligodendrocytes in amyotrophic lateral sclerosis. Nat Neurosci 2013;16:571-579.

899 50. Buchan JR, Kolaitis RM, Taylor JP, Parker R. Eukaryotic stress granules are cleared by 900 autophagy and Cdc48/VCP function. Cell 2013;153:1461-1474.

901 51. Guzhova I, Kislyakova K, Moskaliova O, et al. In vitro studies show that Hsp70 can be released 902 by glia and that exogenous Hsp70 can enhance neuronal stress tolerance. Brain Res 2001;914:6690373.

904 52. Gifondorwa DJ, Robinson MB, Hayes CD, et al. Exogenous delivery of heat shock protein 70 905 increases lifespan in a mouse model of amyotrophic lateral sclerosis. J Neurosci 2007;27:1317390613180.

907 53. Taylor AR, Robinson MB, Gifondorwa DJ, Tytell M, Milligan CE. Regulation of heat shock 908 protein 70 release in astrocytes: role of signaling kinases. Dev Neurobiol 2007;67:1815-1829.

909 54. Sharp FR, Bernaudin M, Bartels M, Wagner KR. Glial expression of heat shock proteins 910 (HSPs) and oxygen-regulated proteins (ORPs). Prog Brain Res 2001;132:427-440.

911 55. Sabatelli M, Zollino M, Conte A, et al. Primary fibroblasts cultures reveal TDP-43 abnormalities 912 in amyotrophic lateral sclerosis patients with and without SOD1 mutations. Neurobiol Aging 913 2015;36:2005 e2005-2005 e2013. 
914 56. Greenberg SA, Pinkus JL, Amato AA. Nuclear membrane proteins are present within rimmed 915 vacuoles in inclusion-body myositis. Muscle Nerve 2006;34:406-416.

916 57. Hatch E, Hetzer M. Breaching the nuclear envelope in development and disease. J Cell Biol 917 2014;205:133-141.

918 58. San Gil R, Ooi L, Yerbury JJ, Ecroyd H. The heat shock response in neurons and astroglia 919 and its role in neurodegenerative diseases. Mol Neurodegener 2017;12:65.

920 59. Verghese J, Abrams J, Wang Y, Morano KA. Biology of the heat shock response and protein 921 chaperones: budding yeast (Saccharomyces cerevisiae) as a model system. Microbiol Mol Biol Rev 922 2012;76:115-158.

923 60. Calderwood SK, Murshid A, Prince T. The shock of aging: molecular chaperones and the heat 924 shock response in longevity and aging--a mini-review. Gerontology 2009;55:550-558.

925

926

927

928

929

930

931

932

933

934

935

936

937

938

939

940

941

942

943

944

945

946

947

948

949

950 
952

953

954

955

956

957

958

959

960

961

962

963

964

965

966

967

968

969

970

971

972

973

974

975

976

977

978

979

980

981

982

983

984

985

986

987

988
A

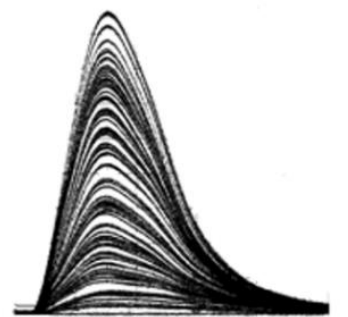

Control

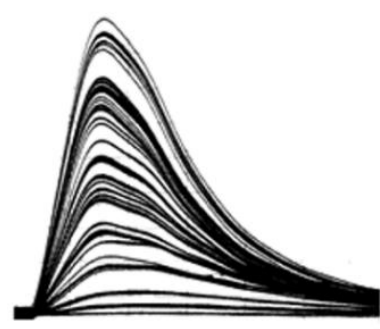

$\mathrm{mVCP}$
B

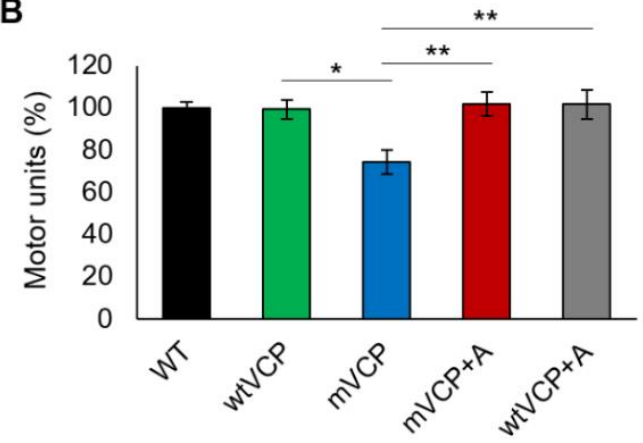

D

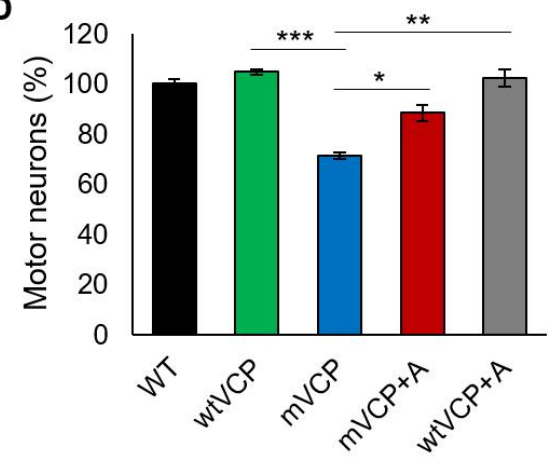

$\mathbf{E}$

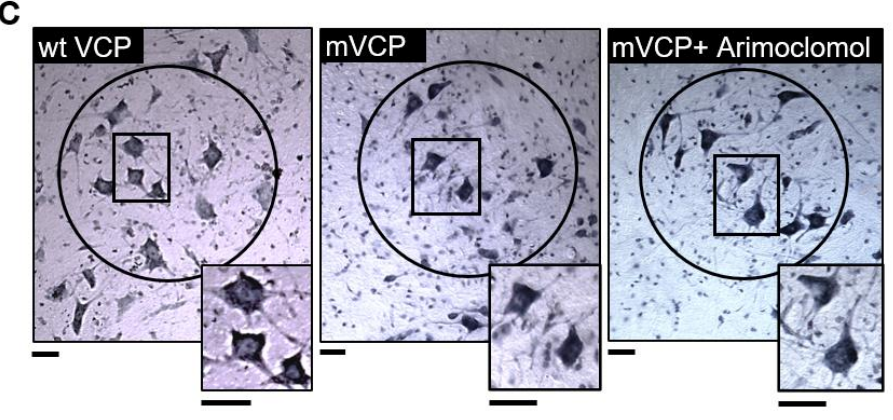

F

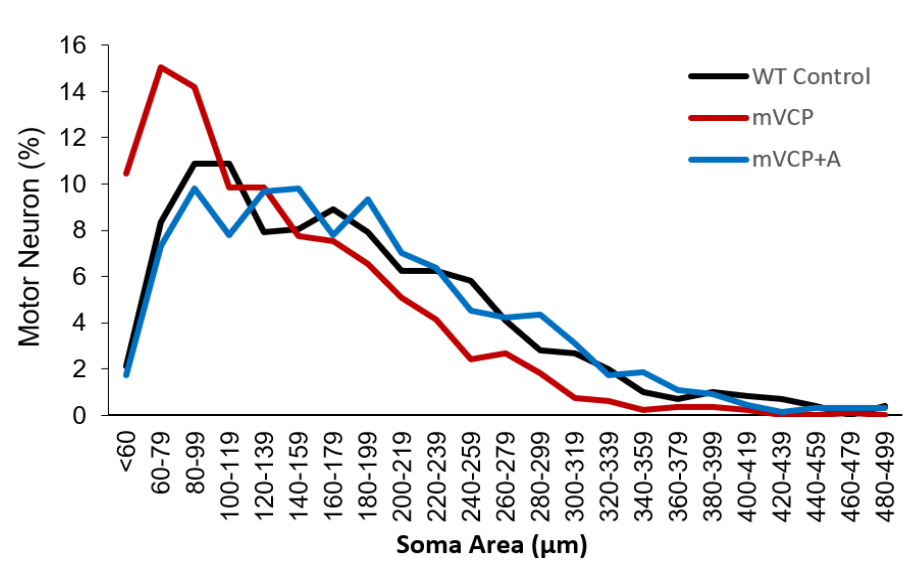

Figure 1. Loss of motor neurons and motor units in mVCP mice is reduced in mice treated with

Arimoclomol. (A) Examples of isometric twitch force traces of the EDL muscle from representative control and mVCP mice. Each increment represents recruitment of a motor unit with increasing nerve stimulation (B) Bar chart shows quantification of motor unit in all experimental groups at 14 months of age. ${ }^{*} \mathrm{p}=0.019,{ }^{* *} \mathrm{p}=0.001$ ( $n=10$ average per experimental group). (C) Nissl stained images of spinal cord sections from the L4 region of wt VCP, mVCP and Arimoclomol treated mVCP mice at 14 months of age. Sciatic pool neurons are circled. Insets show images at higher magnification. Scale bars $=20 \mu \mathrm{m}$. (D) Bar chart showing percentage of motor neurons present in the spinal cord sciatic pool from all experimental groups ${ }^{*} \mathrm{p}=0.029,{ }^{* *} \mathrm{p}=0.0001$ ( $n=5$ animals per group). (E) Bar chart representing the mean motor neuron area across cohorts. ${ }^{* *} \mathrm{p}=0.0001$ ( $n=3$ mice per group). (F) Size distribution graph by total somal area of sciatic pool motor neurons from control, mVCP and Arimoclomol treated mVCP mice (10 images of spinal cord regions L4 and L5, $n=3$ mice per group). 
989

990

991

992

993

994

995

996

997

998

999

1000

1001

1002

1003

1004

1005

1006

1007

1008

1009

1010

1011

1012

1013

1014

1015

1016

1017

1018

1019

1020

1021

1022

1023

1024

1025

1026

1027

1028
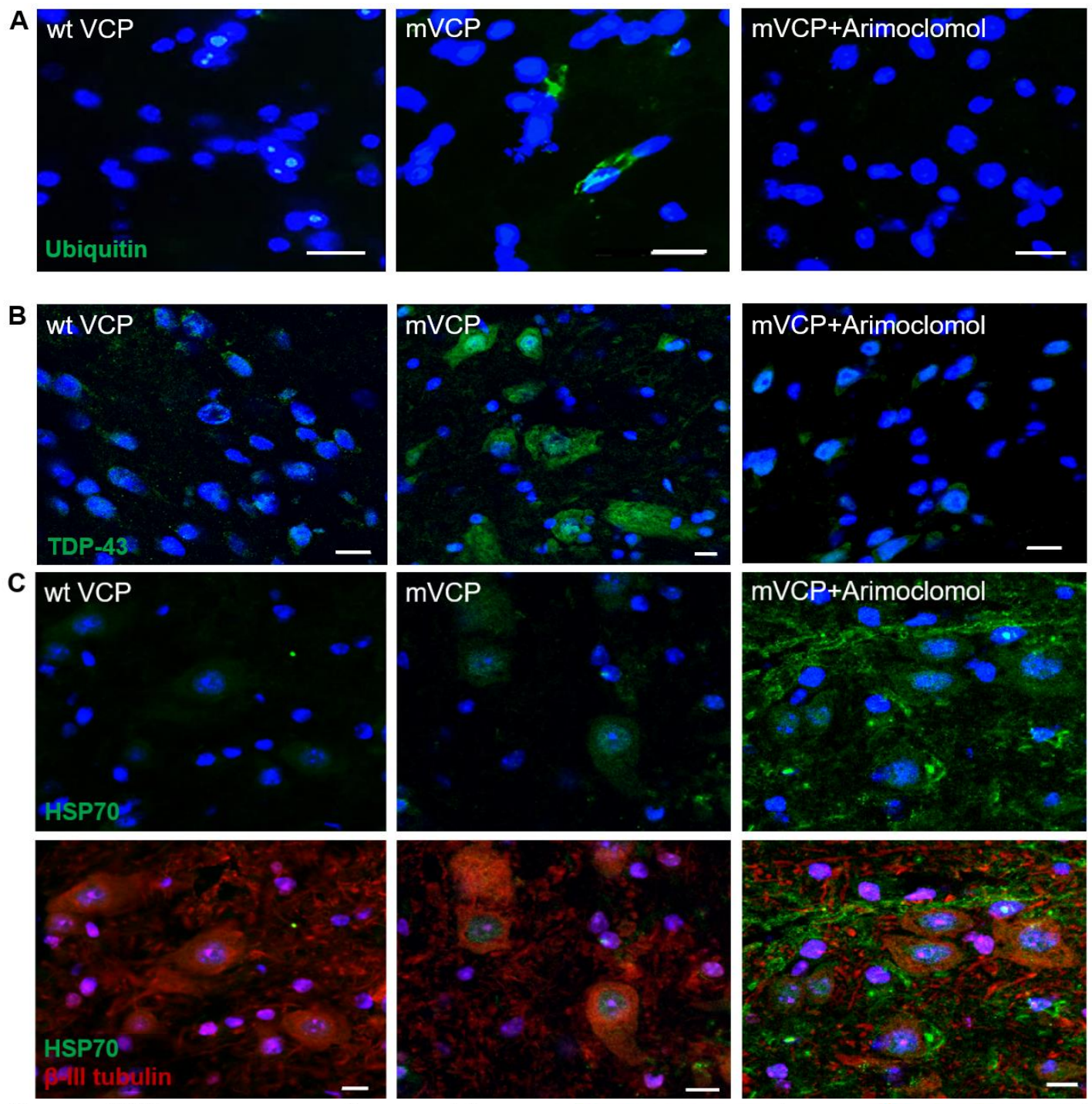

D
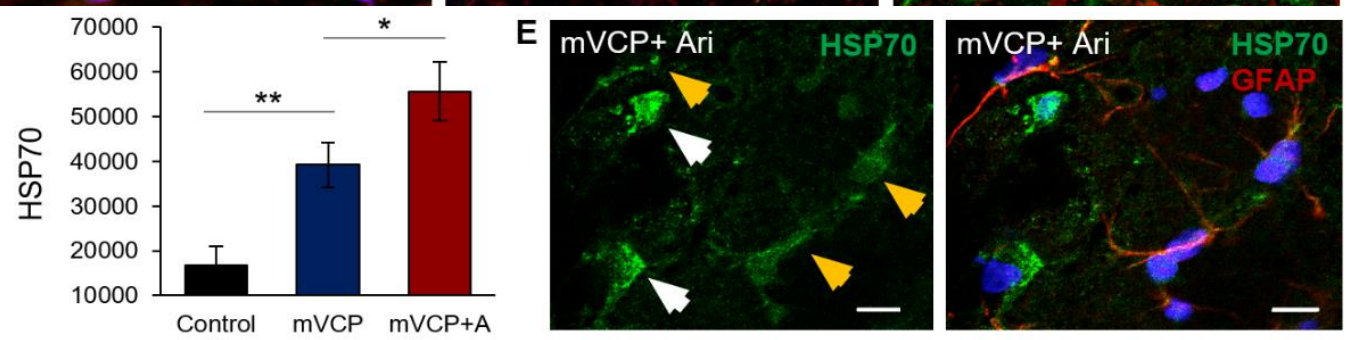

Figure 2. Ubiquitin and TDP-43 pathology in mVCP spinal cord is improved with Arimoclomol and is associated with increased HSP70. Immunofluorescent images of lumber spinal cord sections from wt VCP, mVCP and Arimoclomol treated mVCP mice showing (A) Ubiquitin immunoreactivity in neurons, (B) TDP-43 localisation in sciatic pool neurons and (C) HSP70 expression in spinal cord with and without neuronal marker ( $\beta$-III tubulin, red). (D) Bar chart representing the mean fluorescence intensity (arbitrary values) of HSP70 immunolabelling in individual spinal cord motor neurons using confocal microscopy from each experimental group. $\left.{ }^{*} p=0.044,{ }^{* *} p=0.0056\right)(E)$ HSP70 expression in GFAP co-labelled spinal cord sections with and without the glial marker from an Arimoclomol treated animal. White arrows show GFAP-negative neuronal cells, yellow arrows show GFAP positive glial cells. DAPI labels nuclei (blue). Scale bar $=10 \mu \mathrm{m}$. 

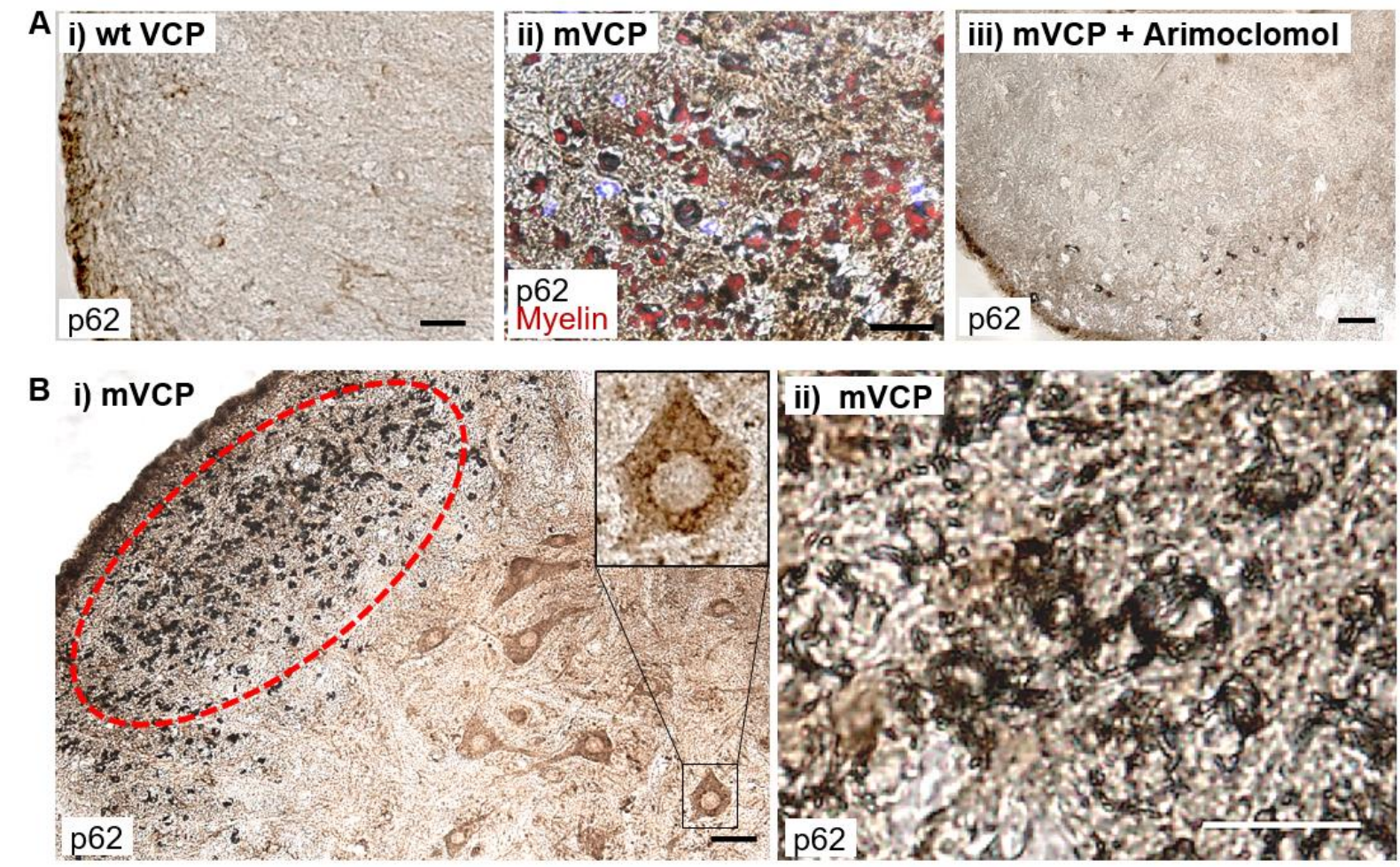

\section{C i) wt VCP}
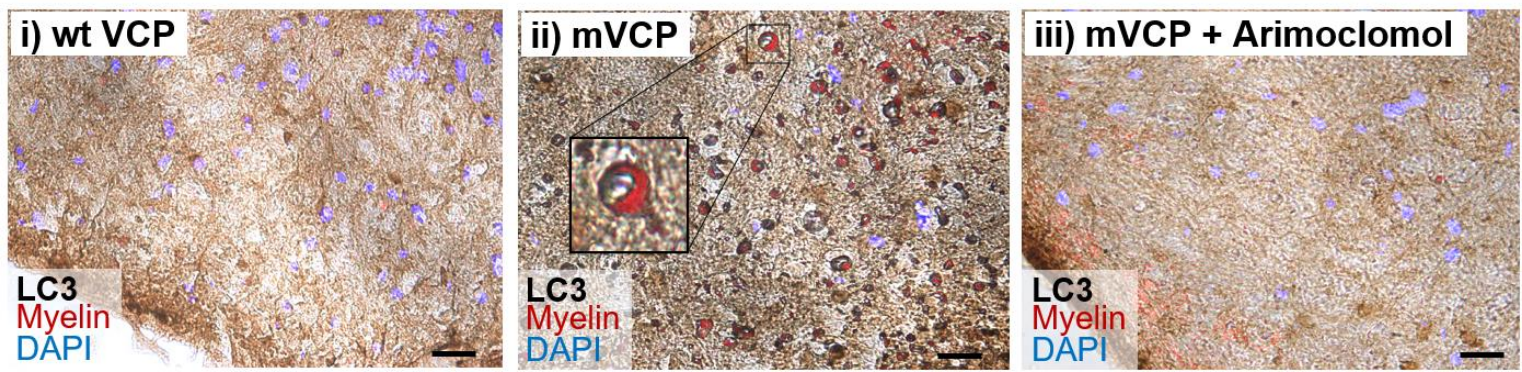

1053

1054

Figure 3. Increased expression of p62 and LC3 in the spinal cord grey and white matter of mVCP

1055 mice is reduced with Arimoclomol treatment. (A) p62 expression in the spinal cord of wt VCP, mVCP and Arimoclomol treated mice. Myelin co-localisation shown in red. (B, i) mVCP spinal cord with increased p62 expression in white and grey matter and aggregated p62 in sciatic pool motor neurons (inset, high magnification image) and oligodendrocytes (ringed in red). ii) High magnification image of increased p62 expression and collapsed myelin sheath in mVCP spinal cord white matter. (C) LC3 expression in mouse spinal cord co-localised with myelin (red). Inset shows high magnification image of a myelinated axon. Scale bar $=10 \mu \mathrm{m}$, DAPI label nuclei (blue).

1062 


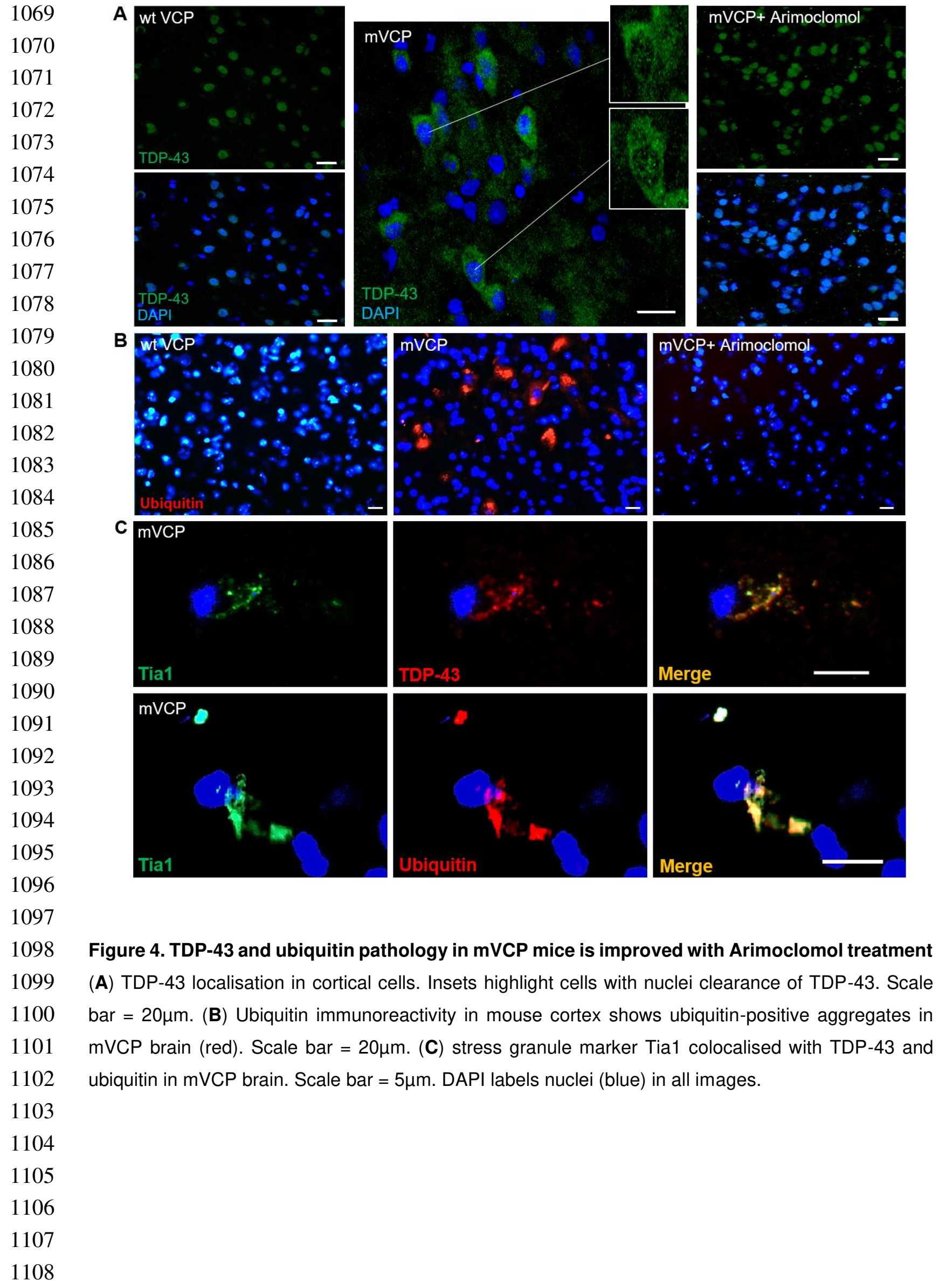



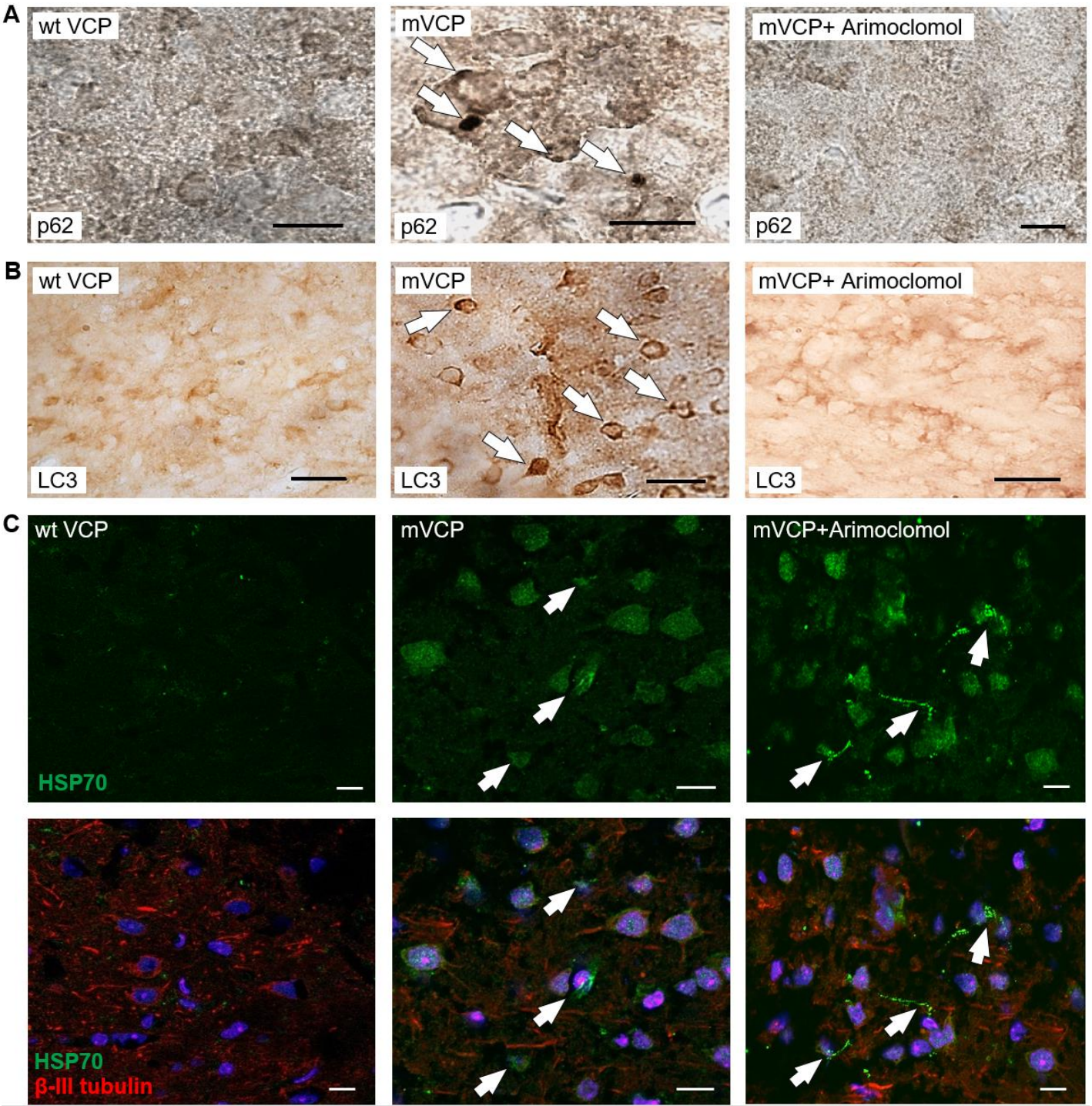

1139

1140

1141

1142

1143

1144

1145

1146

1147

1148

Figure 5. Arimoclomol treatment prevents p62 and LC3 aggregation and enhances HSP70 in mVCP mouse brain. Histological images of (A) p62 expression and (B) LC3 expression in mouse brain sections. White arrows indicate protein aggregates. (C) HSP70 expression in mouse brain with and without neuronal marker ( $\beta$-III tubulin, red) and nuclear marker (DAPI, blue). White arrows indicate glial cells expressing HSP70. Scale bar $=10 \mu \mathrm{m}$. 
A
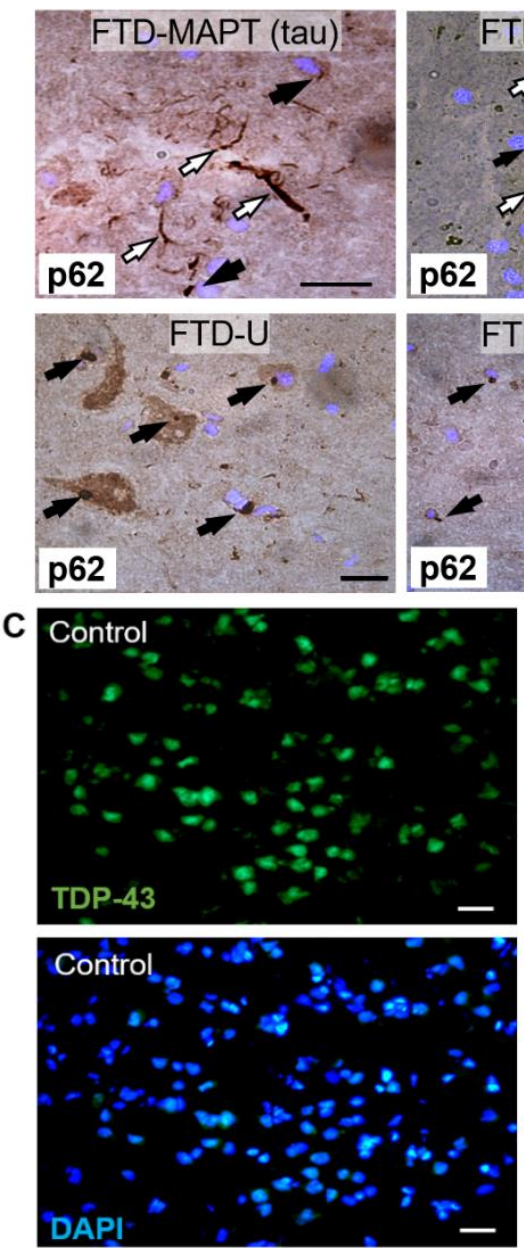
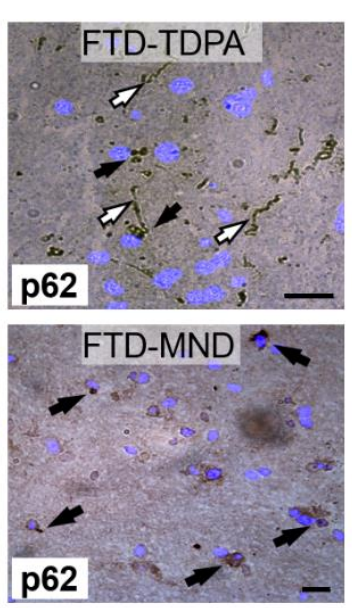

B
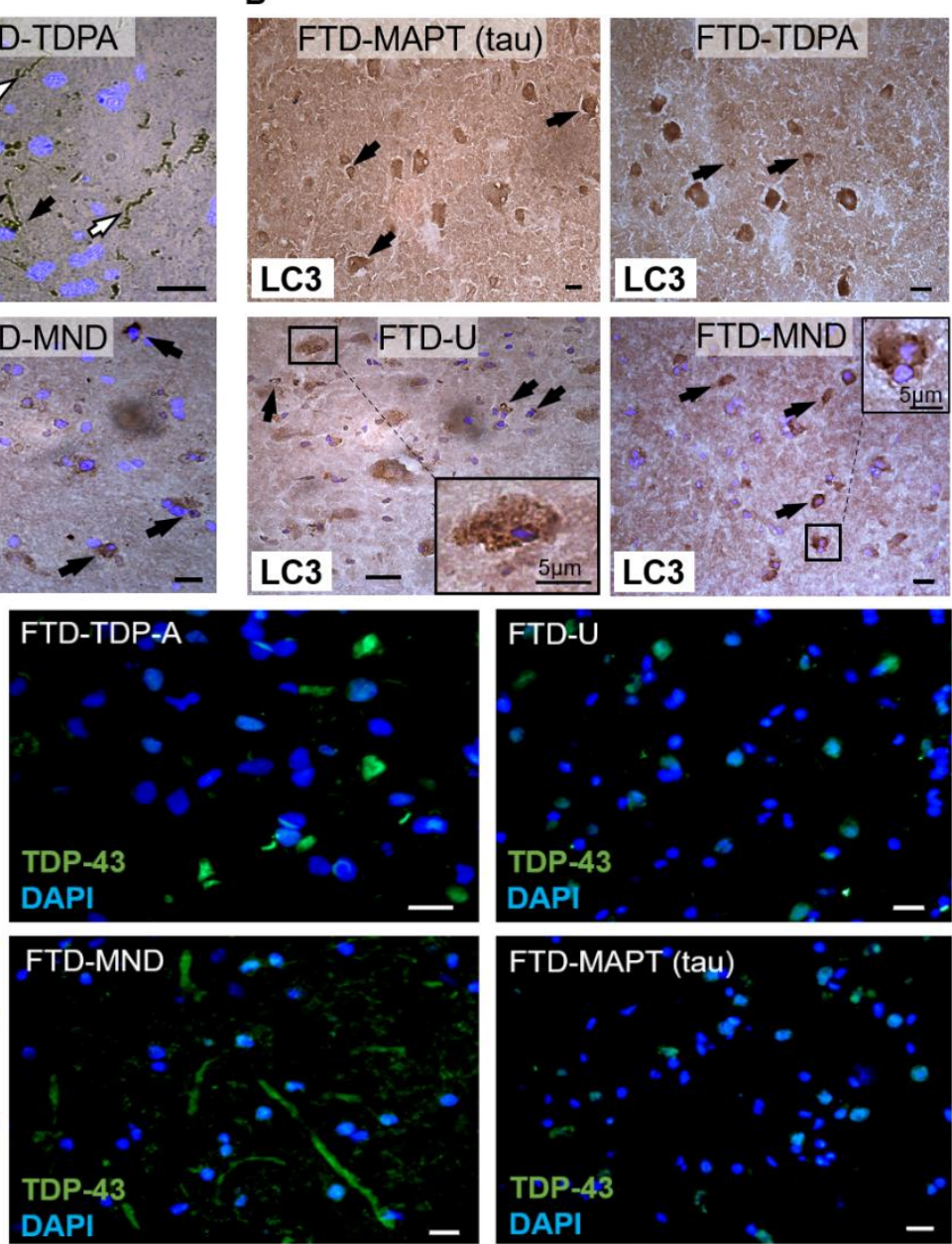

D
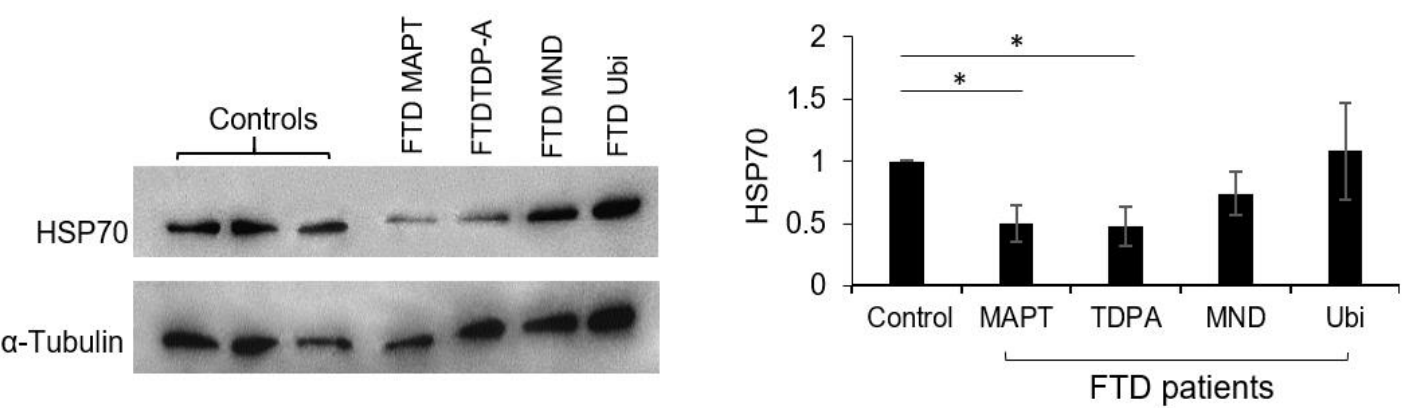

Figure 6. Pathology in brain tissue from FTD patients and their HSP70 expression levels. (A) p62

1160 immunohistochemistry on post-mortem brain cortex from patients with subtypes of FTD shows p62+ 1161 inclusion bodies. White arrows indicate intensely stained neurites and black arrows indicate cytoplasmic 1162 protein aggregates. (B). Increased LC3 expression in neurons of post-mortem FTD brain samples. 1163 Neurons with area of intense positive staining for LC3 in patients with FTD-MAPT and FTD-TDPA. LC31164 positive aggregates observed in neurons from patients with FTD-U and FTD-MND. Insets show 1165 magnification of marked regions. (C) Cytoplasmic TDP-43 mislocalisation was observed in all patient 1166 samples (green), while rarely seen in control tissue. (D) Western blot of HSP70 expression from brain 1167 tissue compared to healthy controls and corresponding density bar chart ( $\left.{ }^{*} p<0.05\right)$. DAPI labels nuclei 1168 (blue). Scale bar $=10 \mu \mathrm{m}$ unless otherwise indicated. 

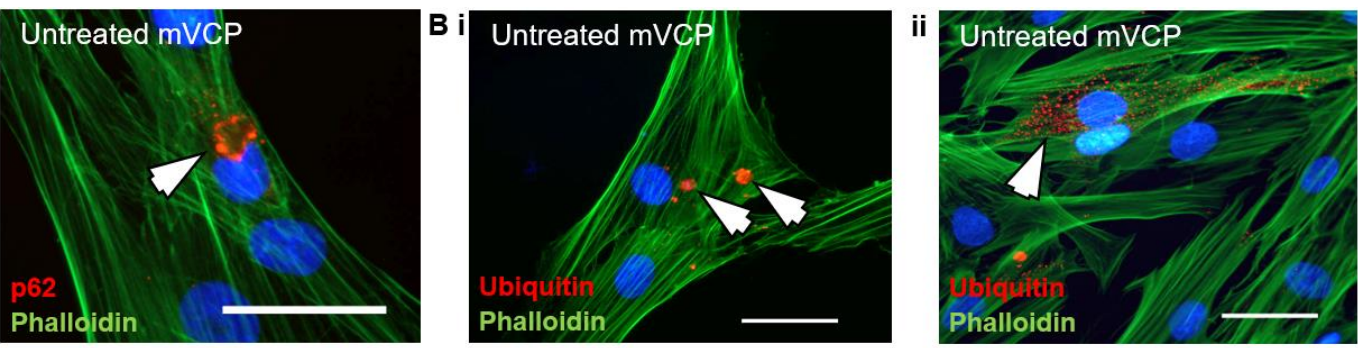

1175

1176

1177

1178

1179

1180

1181

1182

1183

1184

1185

1186

1187

1188
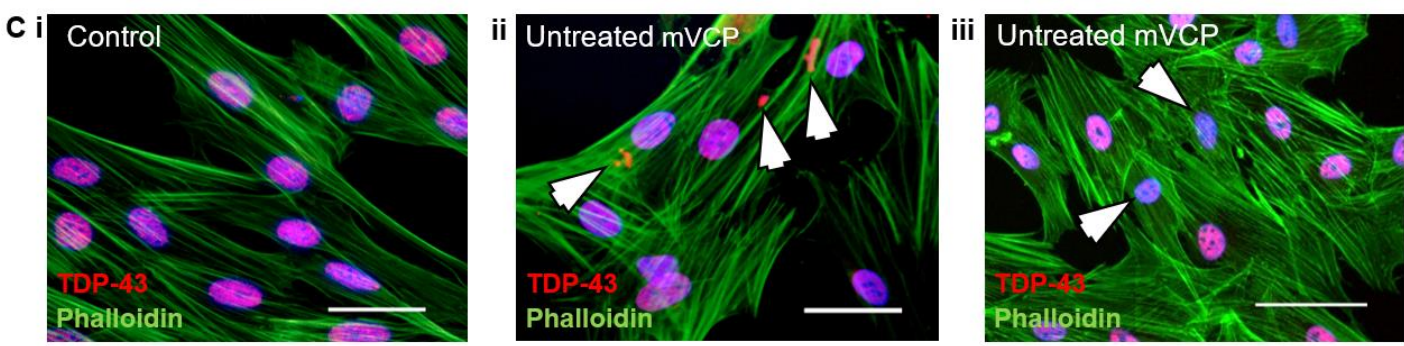

D

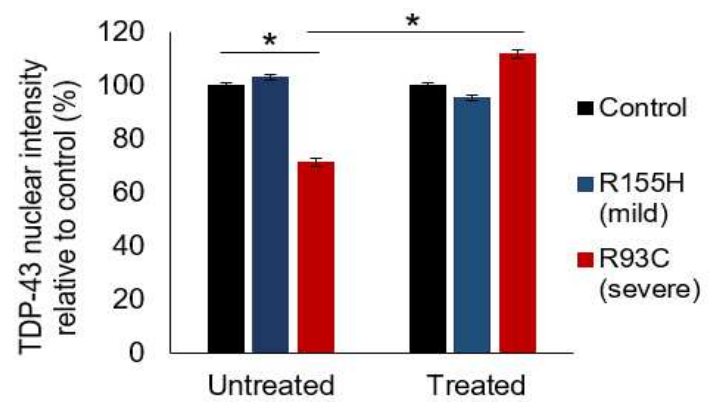

E i

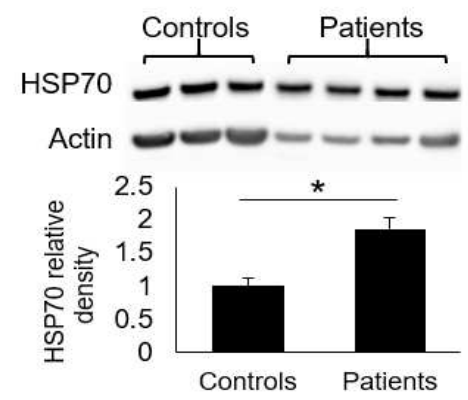

1189

1190

1191

1192

1193

1194

1195

$\mathbf{F}$ i
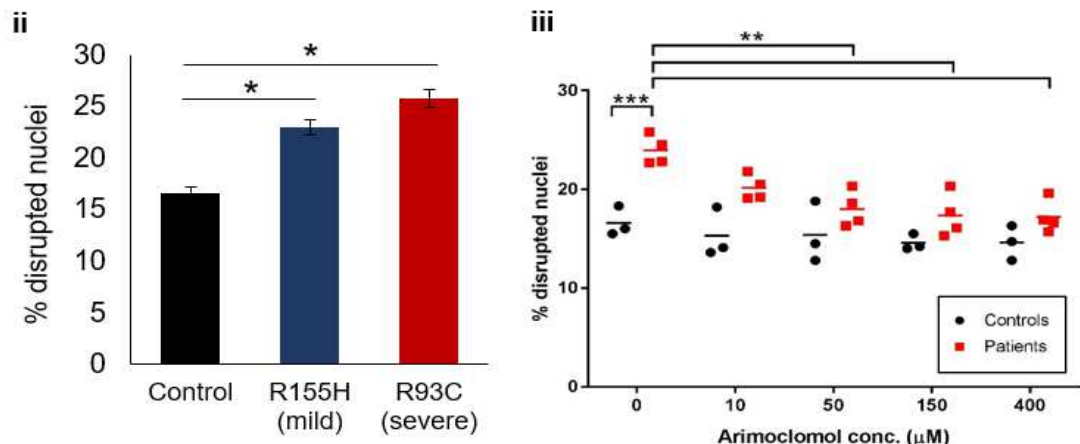

1196

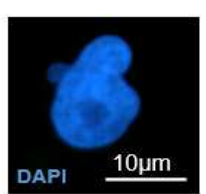

1197

1198

Figure 7. Human mutant VCP patient fibroblasts exhibit pathology ameliorated by Arimoclomol.

1199 Representative immunofluorescent images of untreated mVCP patient fibroblasts demonstrating (A)

1200 p62-positive aggregates, (B) ubiquitin-positive aggregates present as i) large, globular aggregates or

1201 ii) small and diffuse. (C) i) Control image ii) aggregated cytoplasmic TDP-43 and iii) reduced nuclear

1202 abundance of TDP-43, as indicated by white arrows. Scale bar $=20 \mu \mathrm{m}$. (D) Bar chart representation

1203 of TDP-43 nuclear intensity in untreated and $150 \mu \mathrm{M}$ Arimoclomol-treated fibroblasts, 400-500 nuclei

1204 analysed, ${ }^{*} p<0.0001$. (E i) Western blot of HSP70 expression in control and untreated patient fibroblasts

1205 with ii) corresponding density bar chart. ${ }^{*} \mathrm{p}<0.05$ (F) DAPI-labelled fluorescent images of abnormal 1206 nuclear morphology observed in mVCP patient fibroblasts shows i) nuclear herniation and nuclear 1207 fragmentation generating micronuclei. Quantification of percentage disrupted nuclei in (ii) untreated 1208 control and patient fibroblasts, ${ }^{*}<0.0001$ and (iii) fibroblasts treated with increasing concentrations of 1209 Arimoclomol. ${ }^{* *} p<0.01,{ }^{* * *} p<0.001$ 

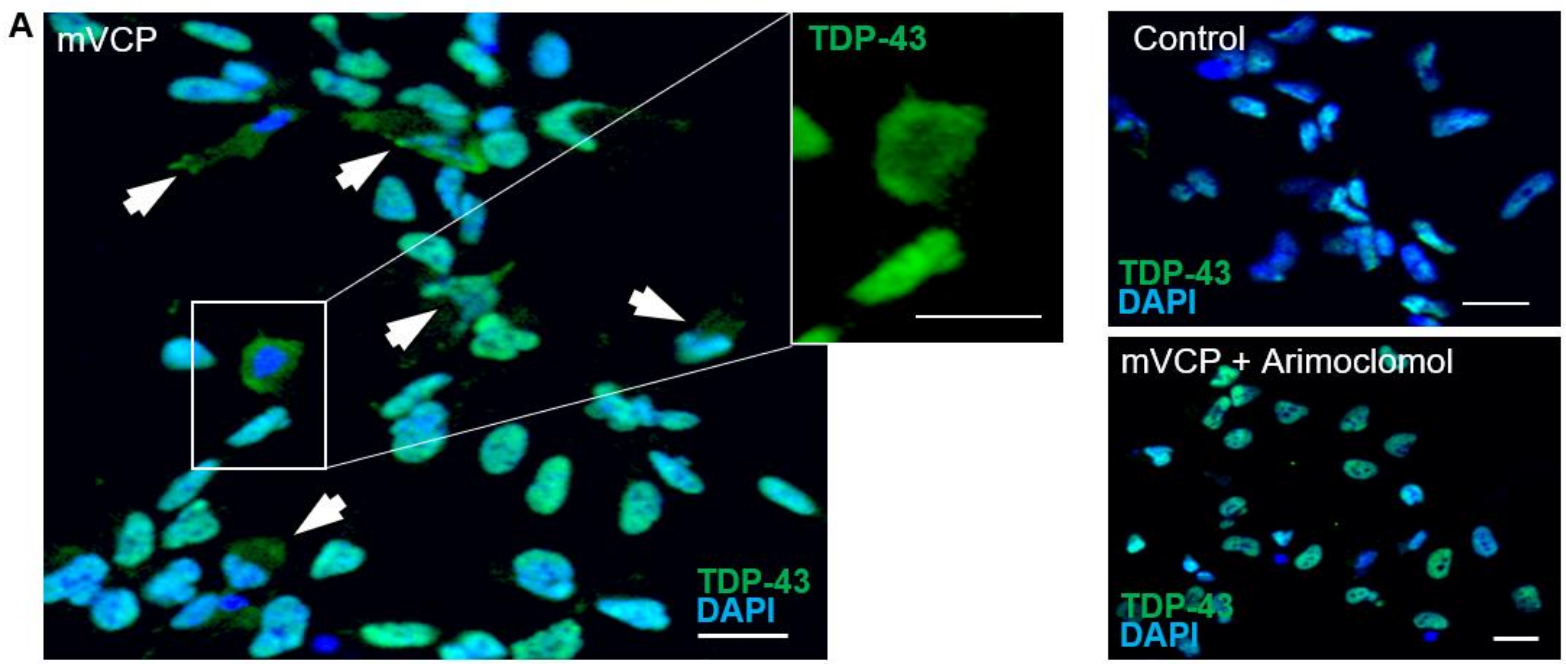

1211

1212

1213

1214

1215

1216

1217

1218

1219

1220
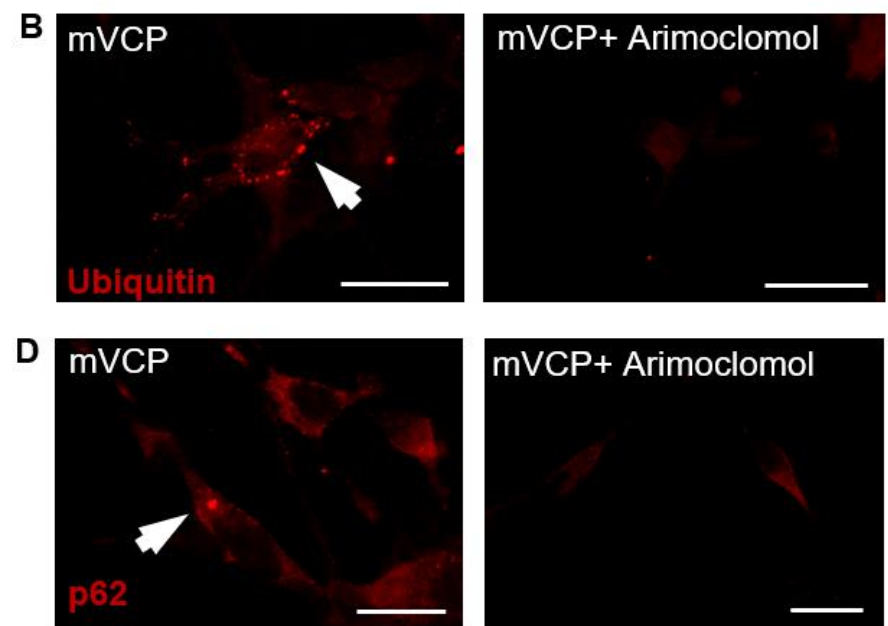

$\mathrm{mVCP}+$ Arimoclomol

E

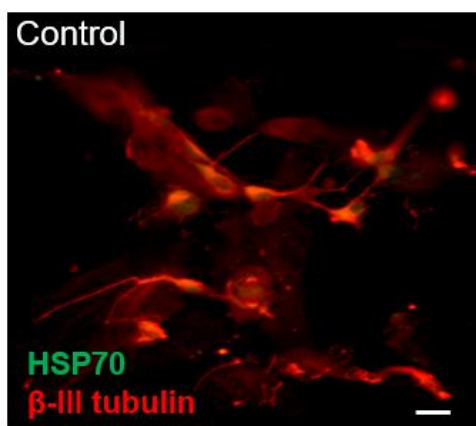

C
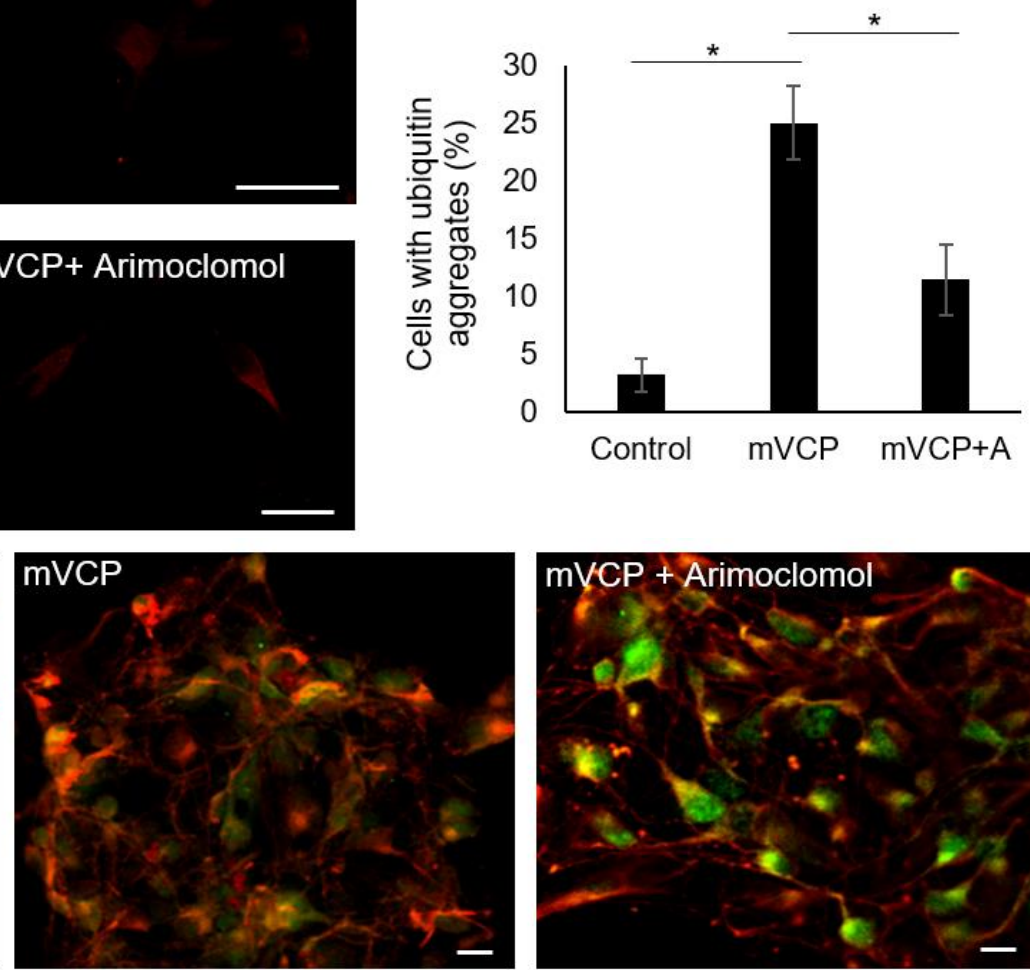

Figure 8. Human mVCP iPSC-derived motor neurons exhibit pathology ameliorated by

Arimoclomol. (A) TDP-43 immunoreactivity shows localisation in control, mVCP and Arimoclomol treated mVCP iPSC motor neuron cultures. Inset shows magnification of cell with nuclear depletion of TDP-43 (B) Fluorescent images of ubiquitin immunoreactivity in mVCP iPSC motor neuron cultures with and without Arimoclomol treatment (C) quantification of cells with ubiquitin-positive aggregates represented as a bar chart. ${ }^{*} p=0.026$ between control and $\mathrm{mVCP}$ and $\mathrm{p}=0.046$ between $\mathrm{mVCP}$ and mVCP+A. (D) p62 immunoreactivity in iPSC motor neurons from Arimoclomol treated and untreated mVCP cultures. (E) immunofluorescent images of HSP70 expression with neuronal marker $\beta$-III tubulin. DAPI labels nuclei (blue). Scale bar $=20 \mu \mathrm{m}$. 


\section{Figures}

A

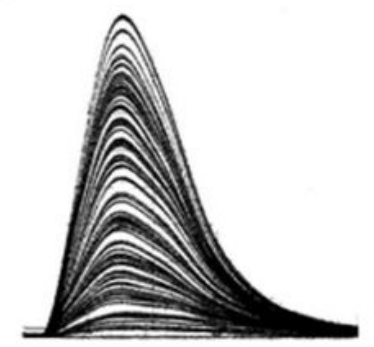

Control

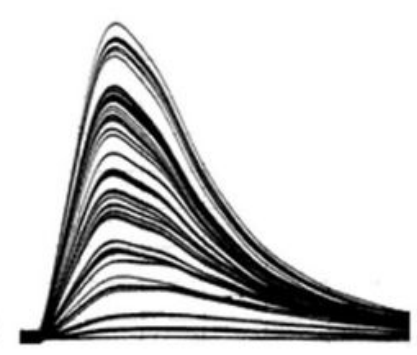

mVCP

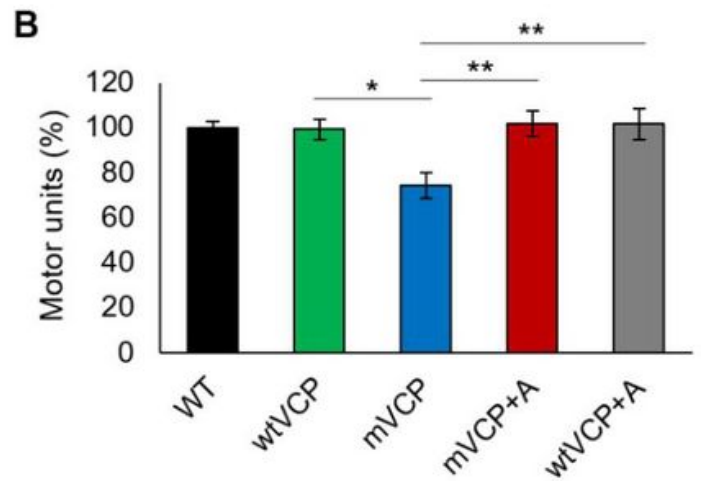

\section{C}
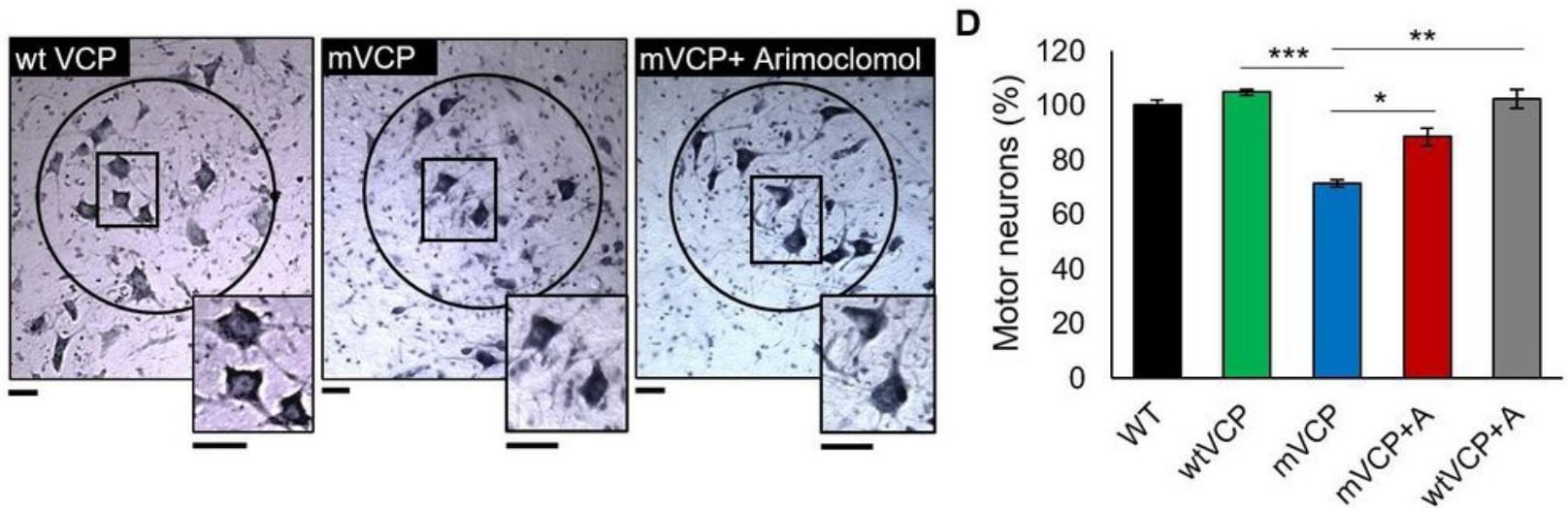

E

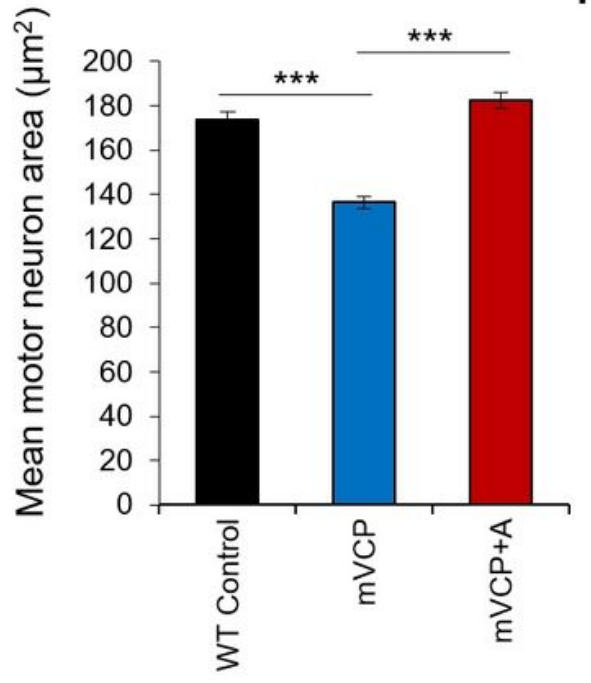

$\mathbf{F}$

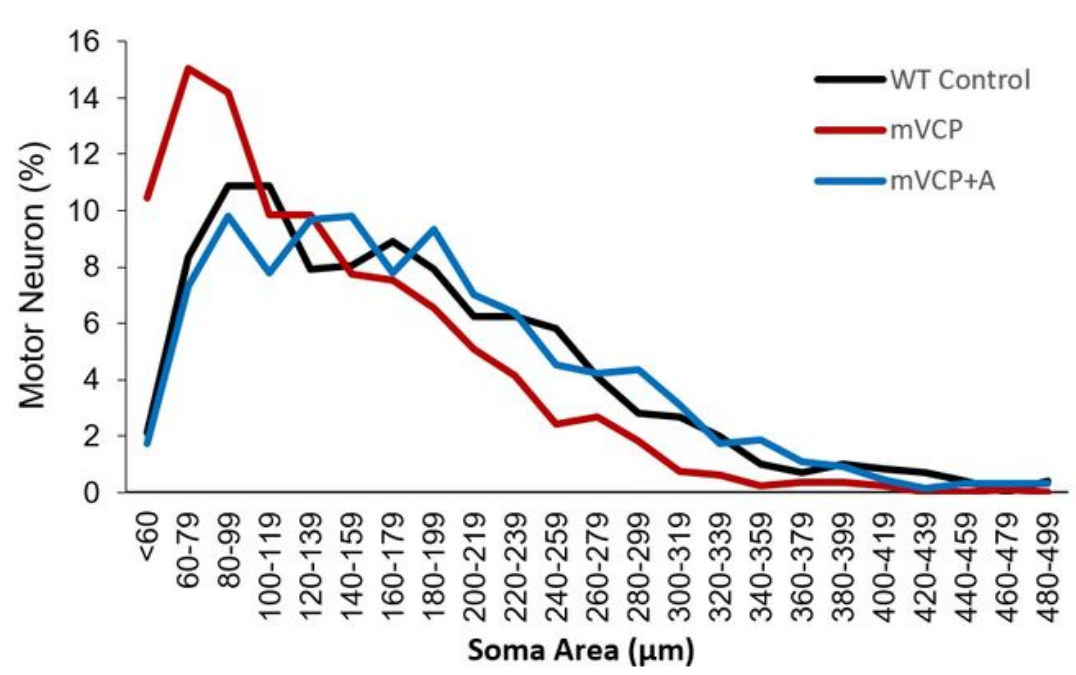

\section{Figure 1}

Loss of motor neurons and motor units in $\mathrm{mVCP}$ mice is reduced in mice treated with Arimoclomol. (A) Examples of isometric twitch force traces of the EDL muscle from representative control and mVCP mice. Each increment represents recruitment of a motor unit with increasing nerve 979 stimulation (B) Bar chart shows quantification of motor unit in all experimental groups at 14 months of age. ${ }^{\star} p=0.019,{ }^{\star \star} p=0.001$ 
( $n=10$ average per experimental group). (C) Nissl stained images of spinal cord sections from the L4 region of wt VCP, mVCP and Arimoclomol treated mVCP mice at 14 months of age. Sciatic pool neurons are circled. Insets show images at higher magnification. Scale bars $=20 \mu \mathrm{m}$. (D) Bar chart showing percentage of motor neurons present in the spinal cord sciatic pool from allexperimental groups ${ }^{*} \mathrm{p}=0.029,{ }^{* \star} \mathrm{p}=0.0001$ ( $\mathrm{n}=5$ animals per group). (E) Bar chart representing the mean motor neuron area across cohorts. ${ }^{* \star} \mathrm{p}=0.0001$ ( $\mathrm{n}=3$ mice per group). (F) Size distribution graph by total somal area of sciatic pool motor neurons from control, $\mathrm{mVCP}$ and Arimoclomol treated mVCP mice (10 images of spinal cord regions $L 4$ and $L 5, n=3$ mice per group). 

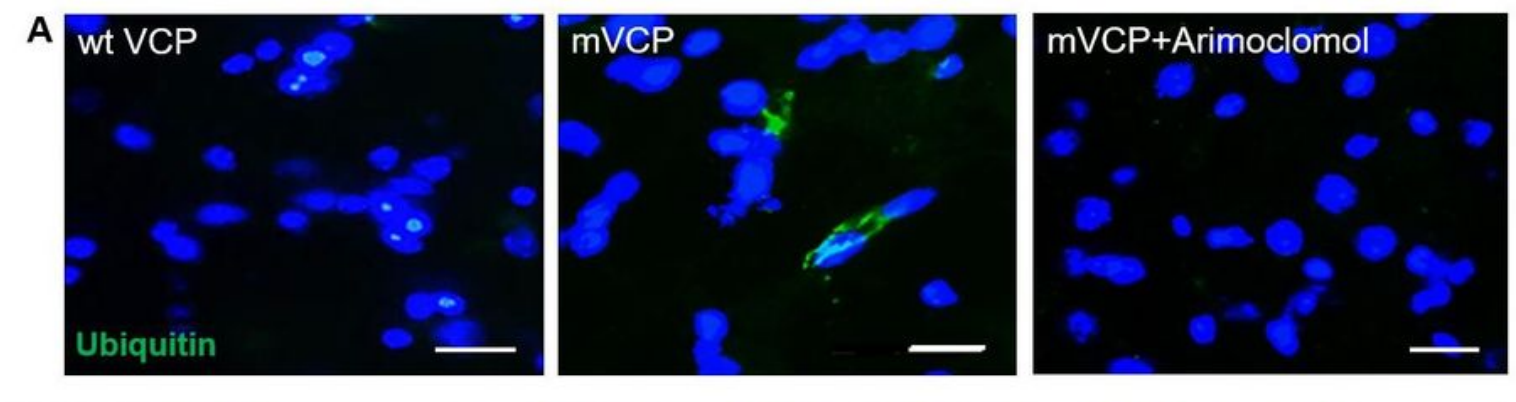

B
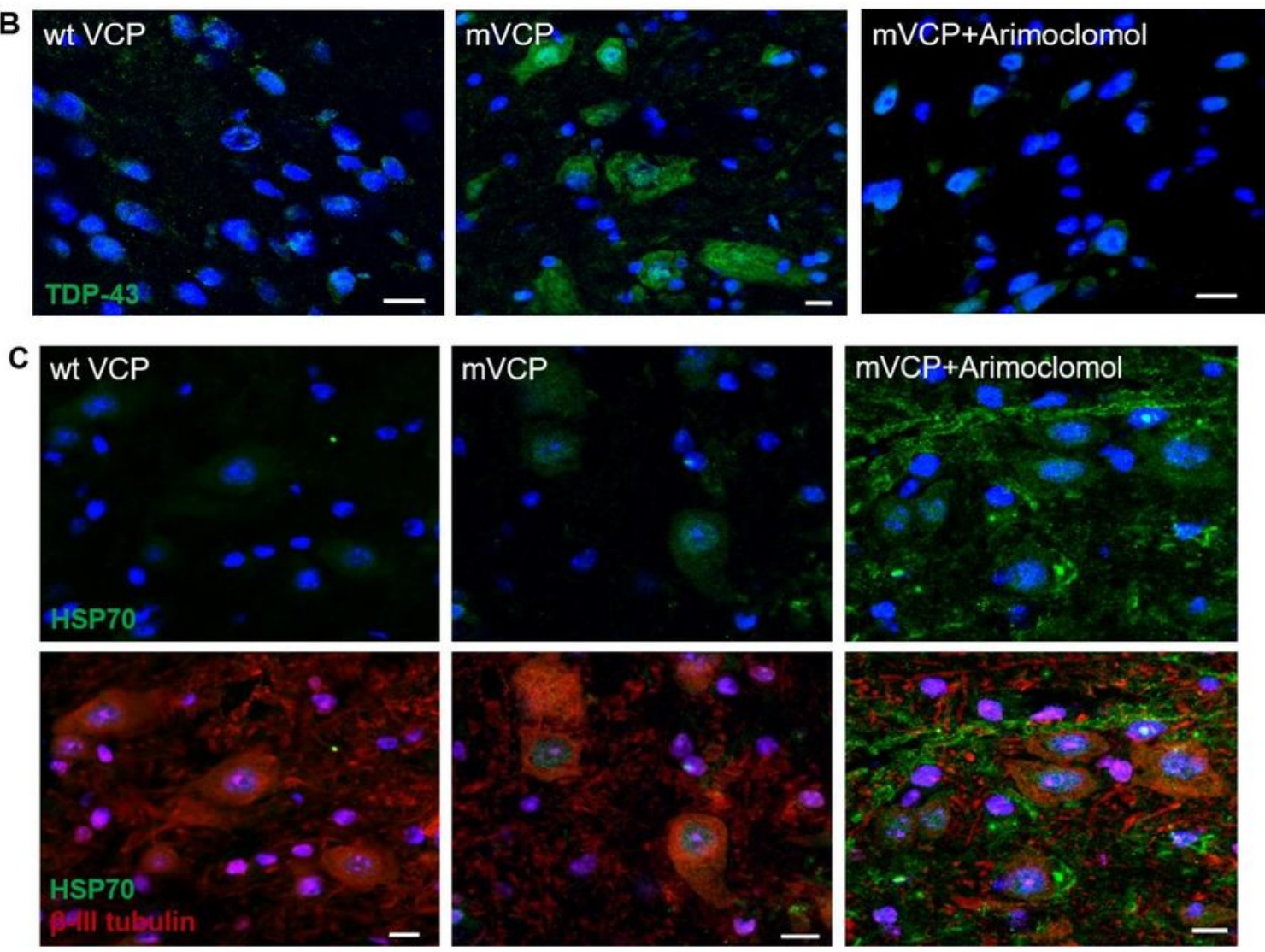

D
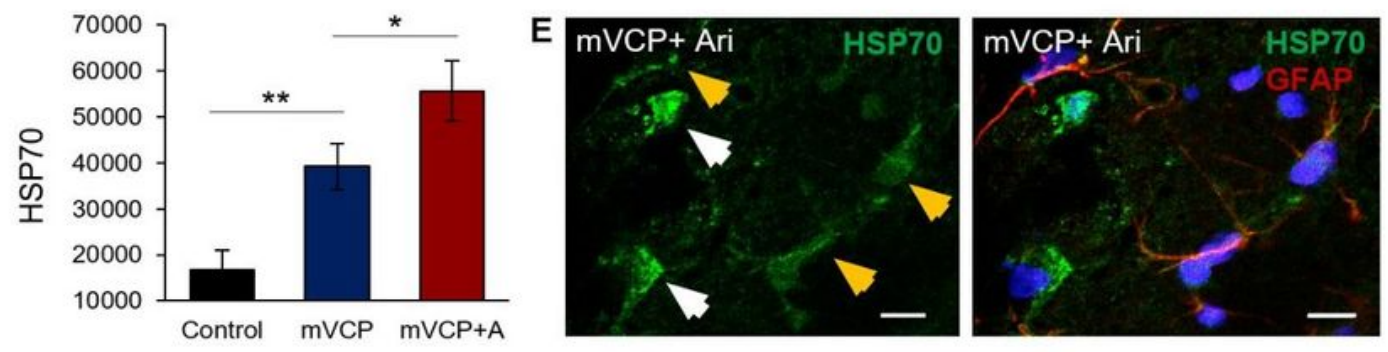

\section{Figure 2}

Ubiquitin and TDP-43 pathology in mVCP spinal cord is improved with Arimoclomol and is associated with increased HSP70. Immunofluorescent images of lumber spinal cord sections from wt VCP, mVCP and Arimoclomol treated mVCP mice showing (A) Ubiquitin immunoreactivity in neurons, (B) TDP-43 localisation in sciatic pool neurons and (C) HSP70 expression in spinal cord with and without neuronal marker ( $\beta$-III tubulin, red). (D) Bar chart representing the mean fluorescence intensity (arbitrary values) of 
HSP70 immunolabelling in individual spinal cord motor neurons using confocal microscopy from each experimental group. $\left.{ }^{*} p=0.044,{ }^{\star *} p=0.0056\right)(E)$ HSP70 expression in GFAP co-labelled spinal cord sections with and without the glial marker from an Arimoclomol treated animal. White arrows show GFAP-negative neuronal cells, yellow arrows show GFAP positive glial cells. 1DAPI labels nuclei (blue). Scale bar $=10 \mu \mathrm{m}$.
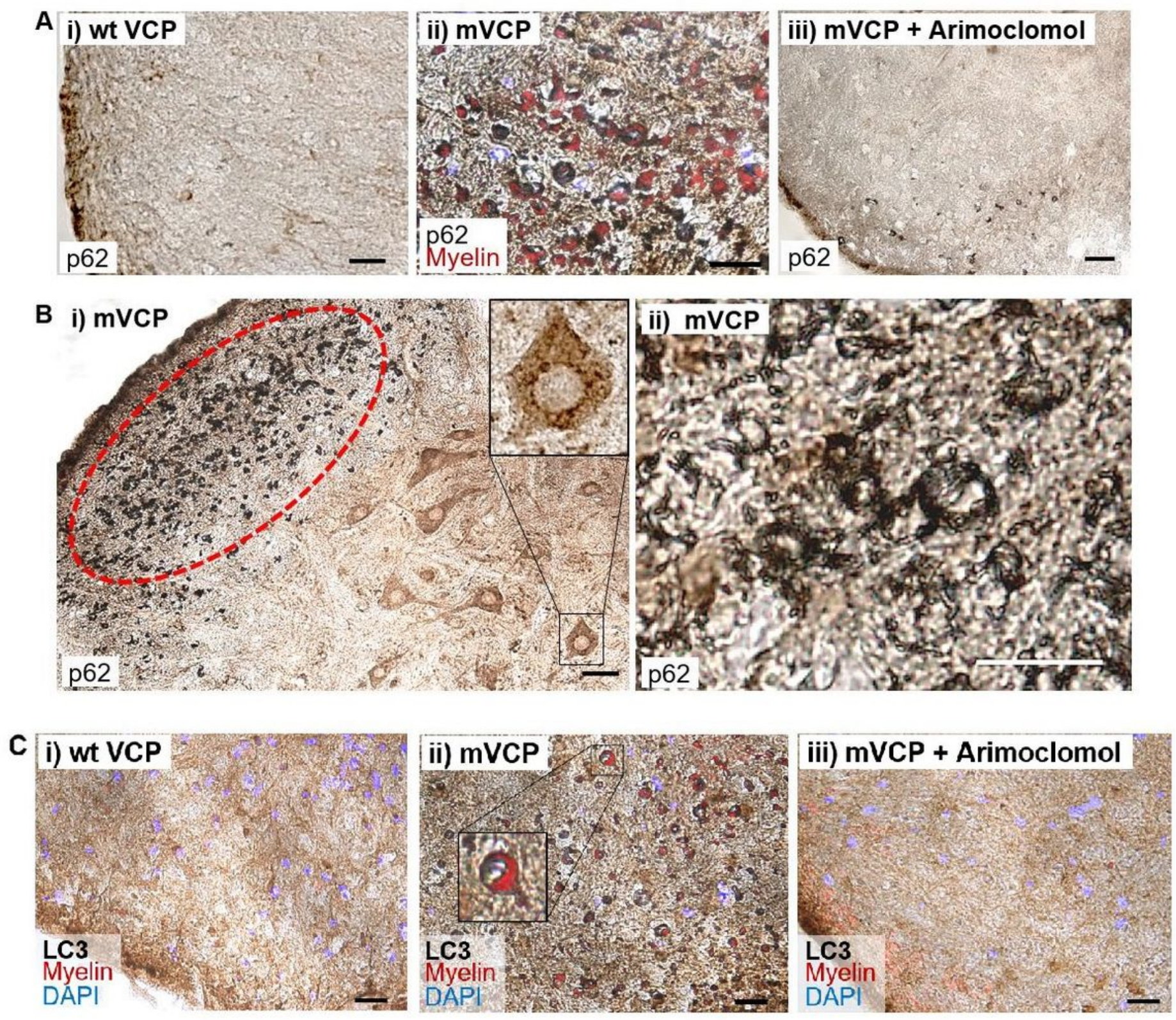

\section{Figure 3}

Increased expression of p62 and LC3 in the spinal cord grey and white matter of mVCP 1054 mice is reduced with Arimoclomol treatment. (A) p62 expression in the spinal cord of wt VCP, mVCP and Arimoclomol treated mice. Myelin co-localisation shown in red. (B, i) mVCP spinal cord with increased p62 expression in white and grey matter and aggregated p62 in sciatic pool motor neurons (inset, high 
magnification image) and oligodendrocytes (ringed in red). ii) High magnification image of increased p62 expression and collapsed myelin sheath in mVCP spinal cord white matter. (C) LC3 expression in mouse spinal cord co-localised with myelin (red). Inset shows high magnification image of a myelinated axon. Scale bar $=10 \mu \mathrm{m}$, DAPI label nuclei (blue).
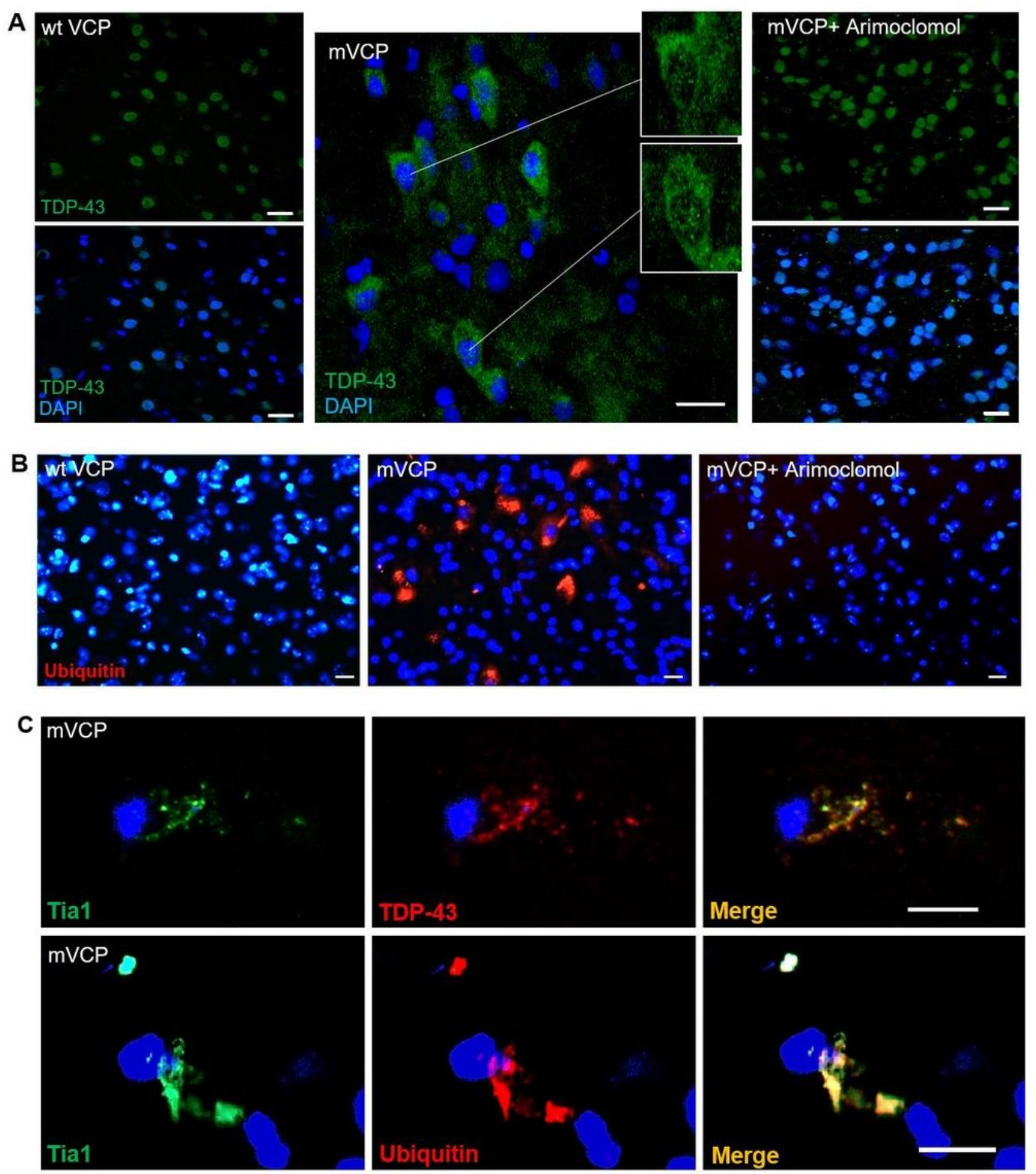

Figure 4 
TDP-43 and ubiquitin pathology in mVCP mice is improved with Arimoclomol treatment (A) TDP-43 localisation in cortical cells. Insets highlight cells with nuclei clearance of TDP-43. Scale bar $=20 \mu \mathrm{m}$. (B) Ubiquitin immunoreactivity in mouse cortex shows ubiquitin-positive aggregates in mVCP brain (red). Scale bar $=20 \mu \mathrm{m}$. (C) stress granule marker Tia1 colocalised with TDP-43 and ubiquitin in mVCP brain. Scale bar $=5 \mu \mathrm{m}$. DAPI labels nuclei (blue) in all images.
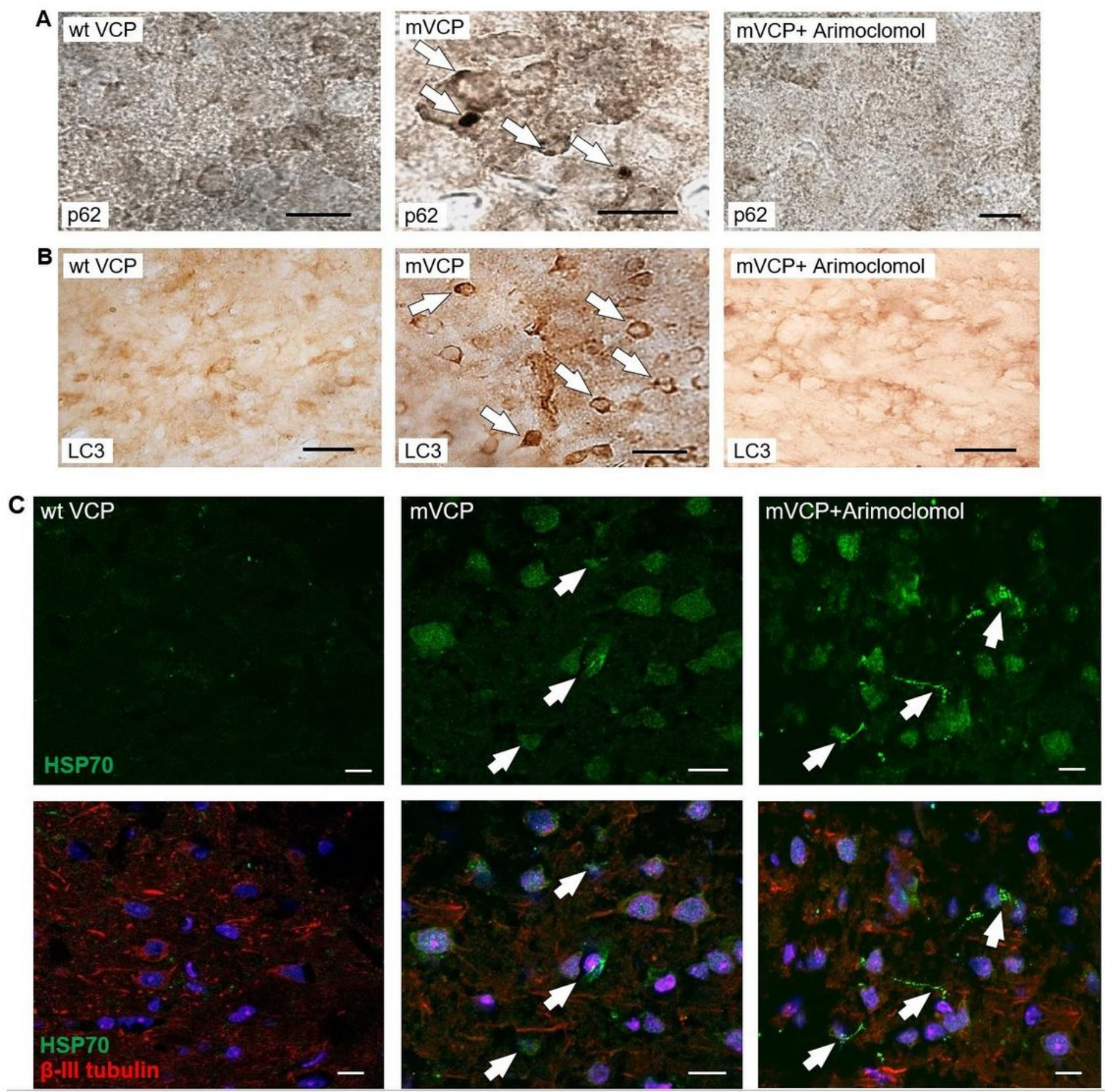

Figure 5 
Arimoclomol treatment prevents p62 and LC3 aggregation and enhances HSP70 in mVCP mouse brain. Histological images of (A) p62 expression and (B) LC3 expression in mouse brain sections. White arrows indicate protein aggregates. (C) HSP70 expression in mouse brain with and without neuronal marker ( $\beta$-III tubulin, red) and nuclear marker (DAPI, blue). White arrows indicate glial cells expressing HSP70. Scale bar $=10 \mu \mathrm{m}$.
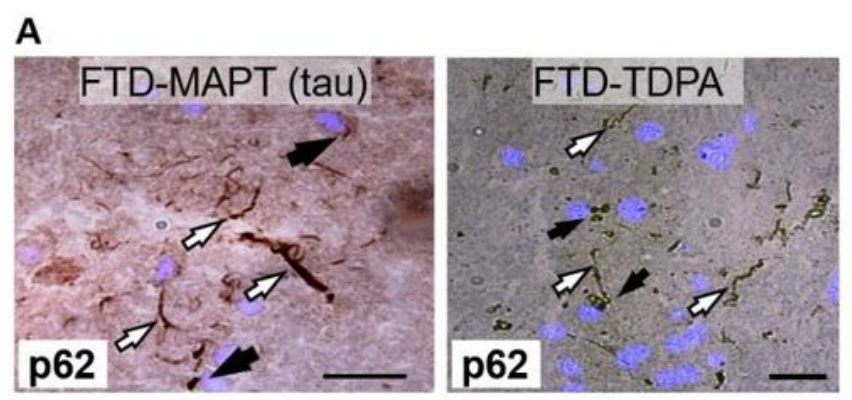

B
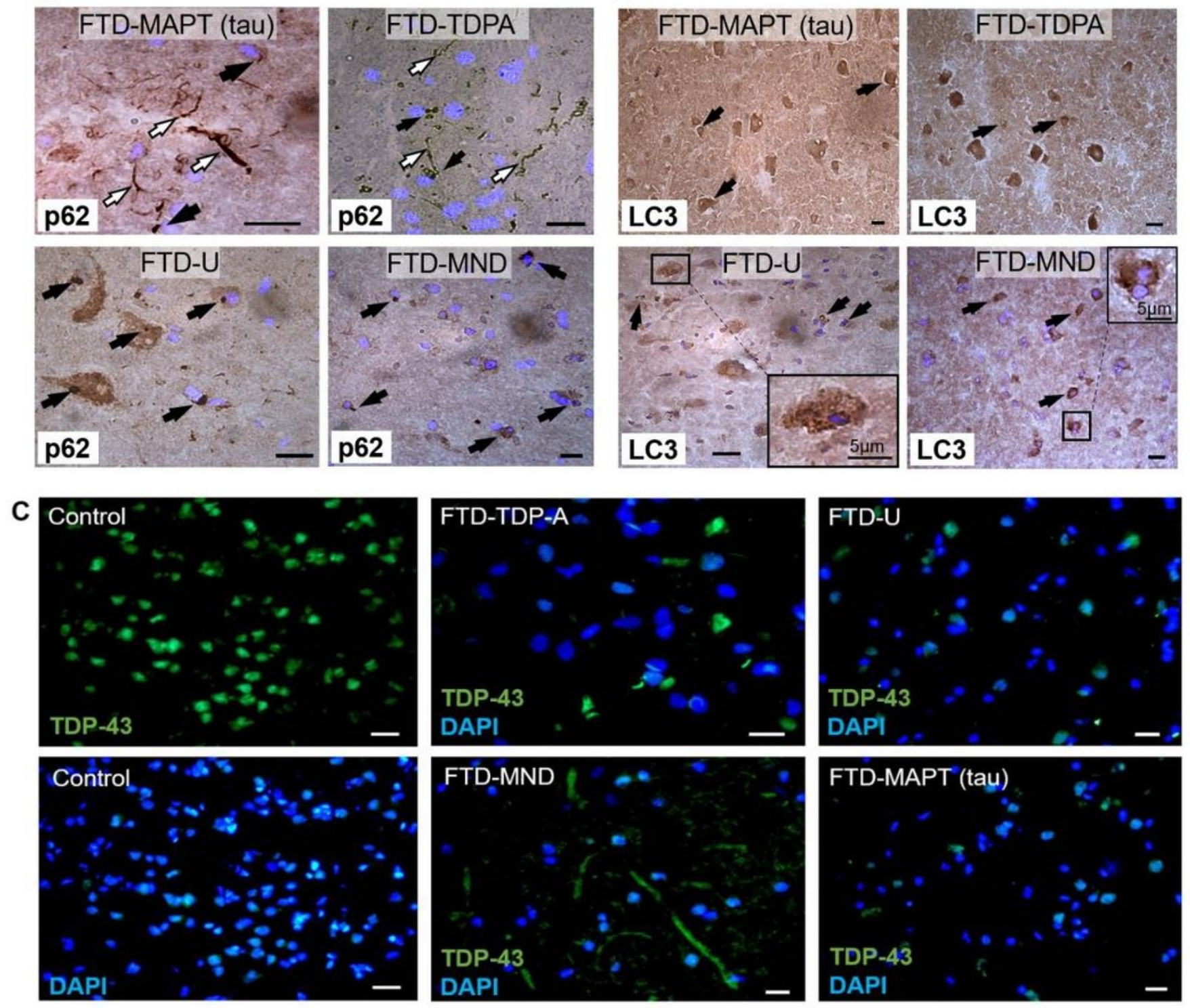

D
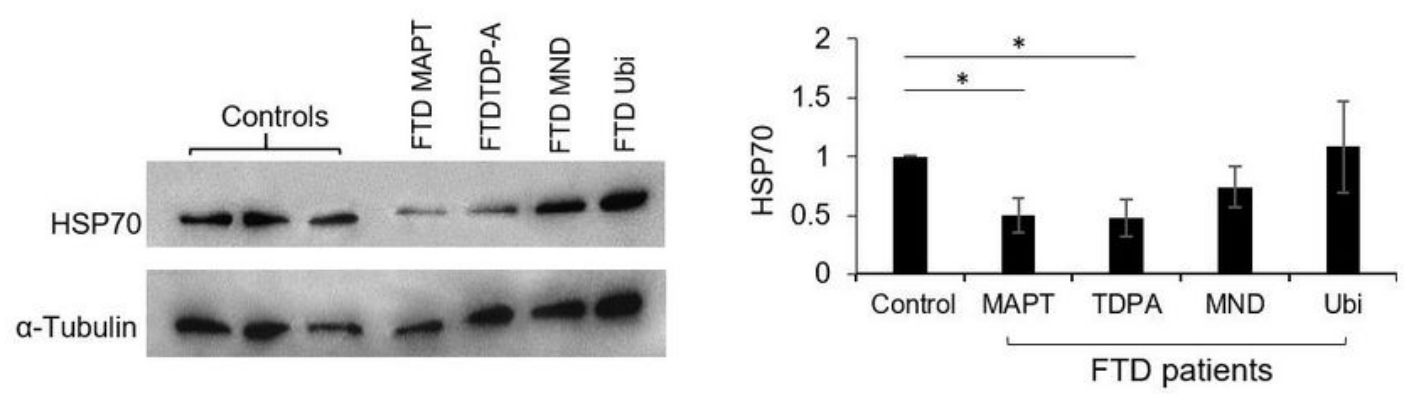

Figure 6 
Pathology in brain tissue from FTD patients and their HSP70 expression levels. (A) p62 immunohistochemistry on post-mortem brain cortex from patients with subtypes of FTD shows p62+ inclusion bodies. White arrows indicate intensely stained neurites and black arrows indicate cytoplasmic protein aggregates. (B). Increased LC3 expression in neurons of post-mortem FTD brain samples. Neurons with area of intense positive staining for LC3 in patients with FTD-MAPT and FTD-TDPA. LC3positive aggregates observed in neurons from patients with FTD-U and FTD-MND. Insets show magnification of marked regions. (C) Cytoplasmic TDP-43 mislocalisation was observed in all patient samples (green), while rarely seen in control tissue. (D) Western blot of HSP70 expression from brain tissue compared to healthy controls and corresponding density bar chart $\left({ }^{*} p<0.05\right)$. DAPI labels nuclei (blue). Scale bar $=10 \mu \mathrm{m}$ unless otherwise indicated. 

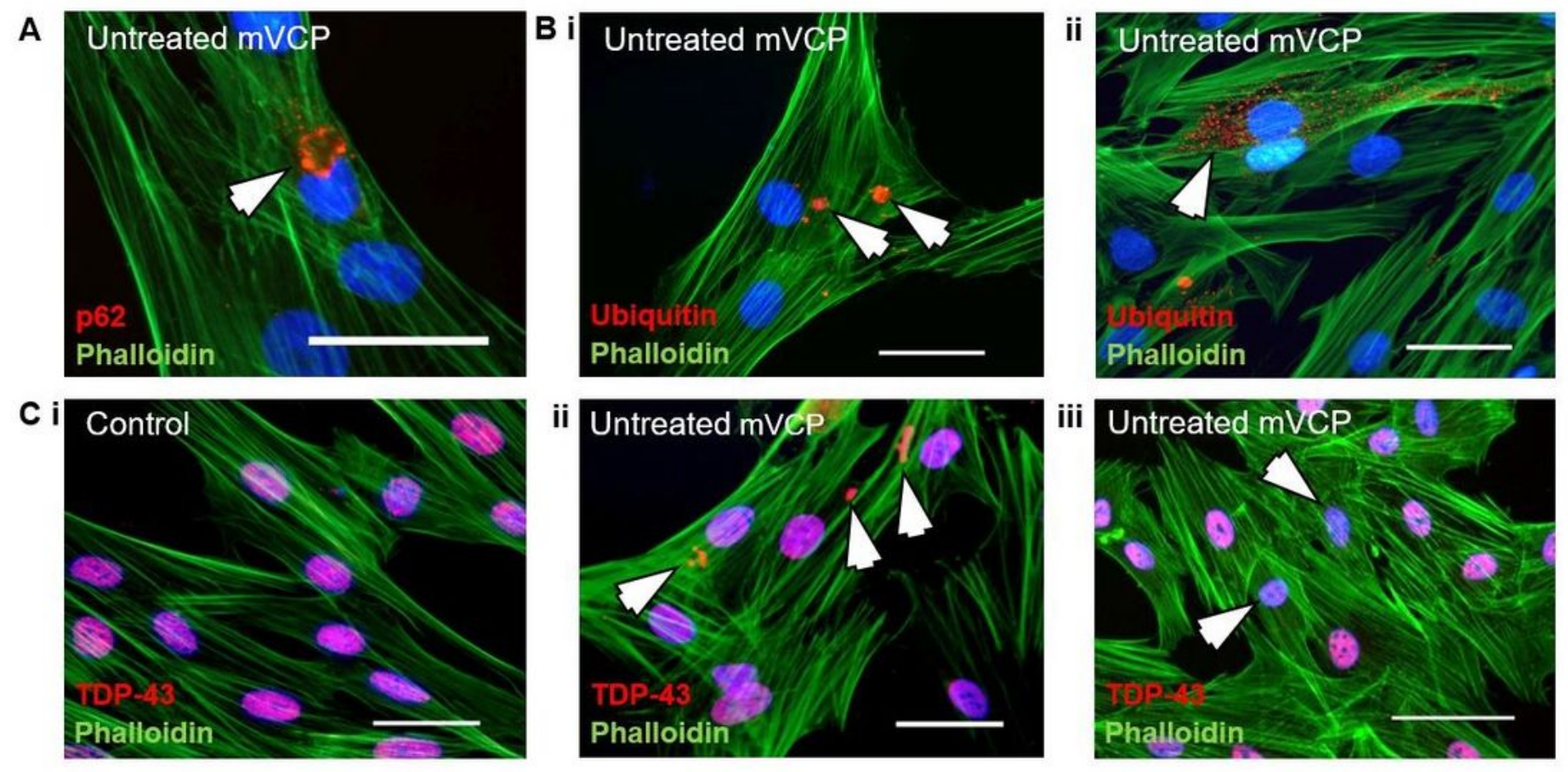

D

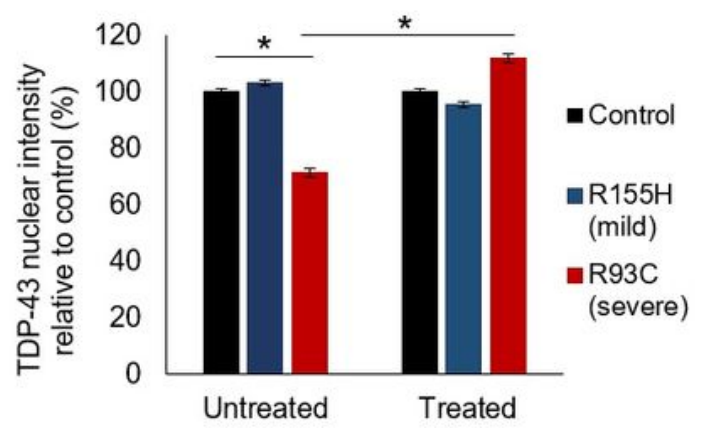

$\mathbf{F i}$
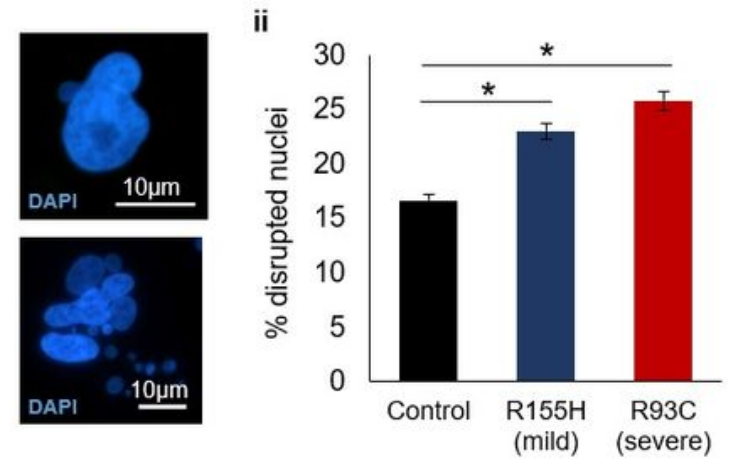

E i

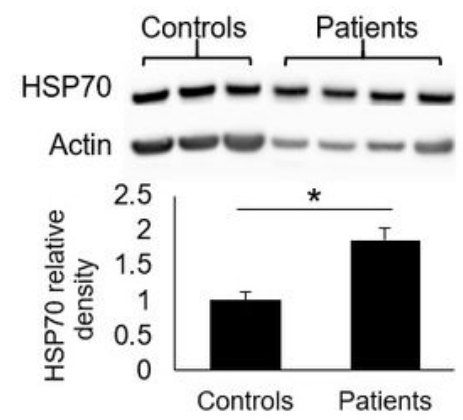

iii

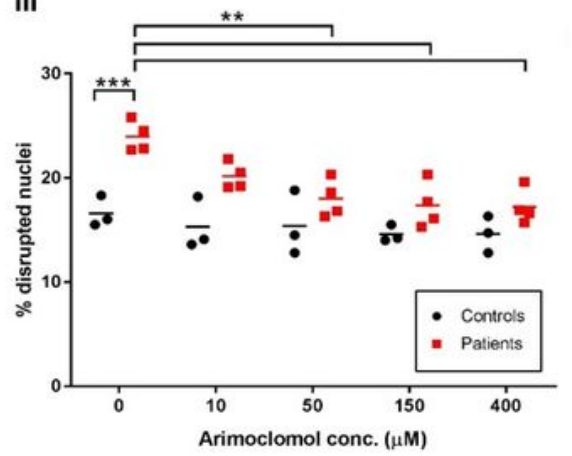

\section{Figure 7}

Human mutant VCP patient fibroblasts exhibit pathology ameliorated by Arimoclomol.Representative immunofluorescent images of untreated mVCP patient fibroblasts demonstrating (A) p62-positive aggregates, (B) ubiquitin-positive aggregates present as i) large, globular aggregates or ii) small and diffuse. (C) i) Control image ii) aggregated cytoplasmic TDP-43 and iii) reduced nuclear abundance of TDP-43, as indicated by white arrows. Scale bar $=20 \mu \mathrm{m}$. (D) Bar chart representation of TDP-43 nuclear 
intensity in untreated and $150 \mu \mathrm{M}$ Arimoclomol-treated fibroblasts, 400-500 nuclei analysed, ${ }^{\star} \mathrm{p}<0.0001$. (E i) Western blot of HSP70 expression in control and untreated patient fibroblasts with ii) corresponding density bar chart. ${ }^{\star} p<0.05$ (F) DAPI-labelled fluorescent images of abnormal nuclear morphology observed in $\mathrm{mVCP}$ patient fibroblasts shows i) nuclear herniation and nuclear fragmentation generating micronuclei. Quantification of percentage disrupted nuclei in (ii) untreated control and patient fibroblasts, ${ }^{*}<0.0001$ and (iii) fibroblasts treated with increasing concentrations of Arimoclomol. ${ }^{*} p<0.01, * \star *$ $p<0.001$
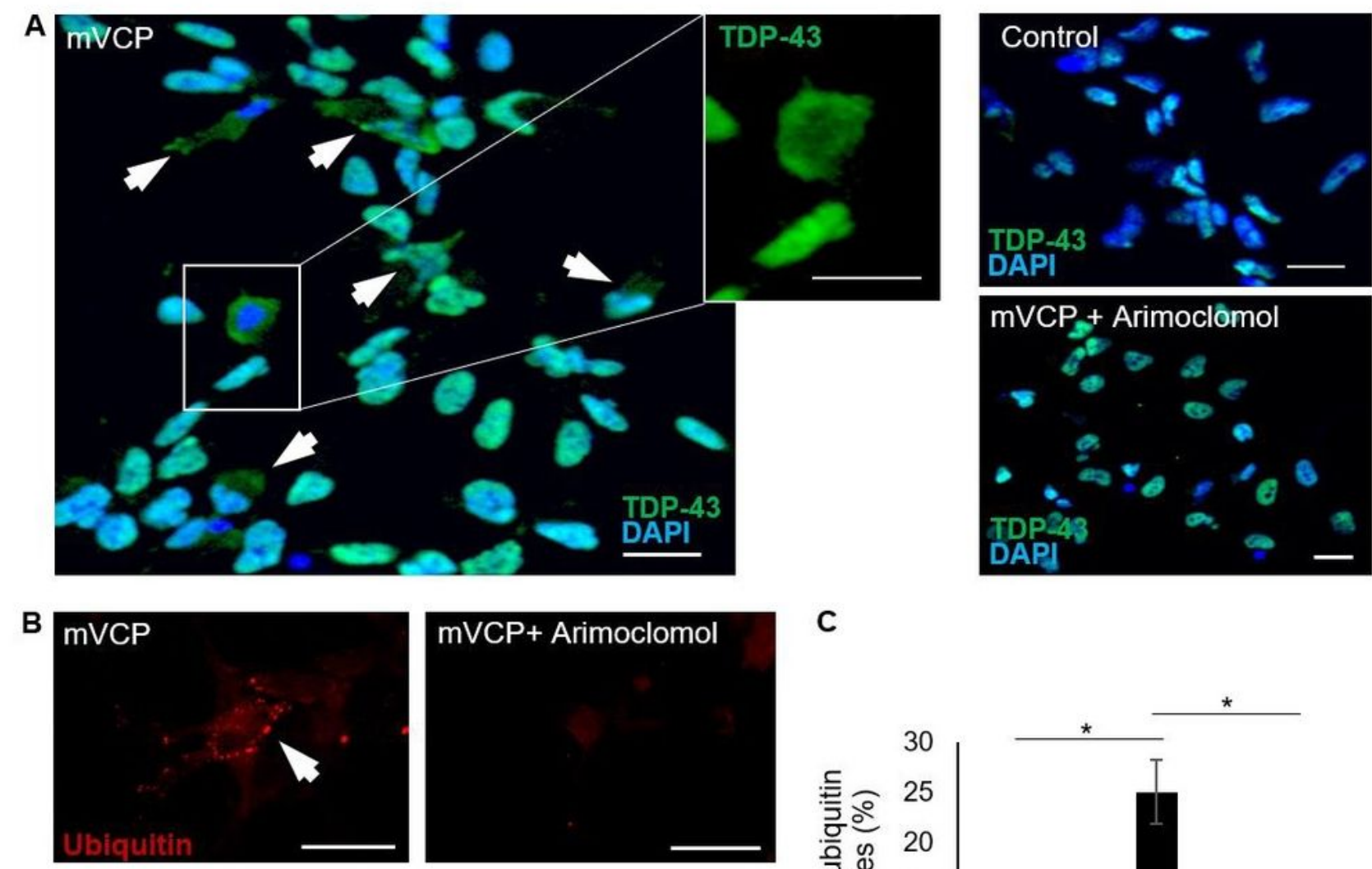

C

D

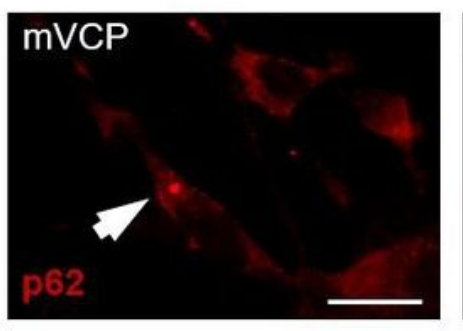

mVCP+ Arimoclomol
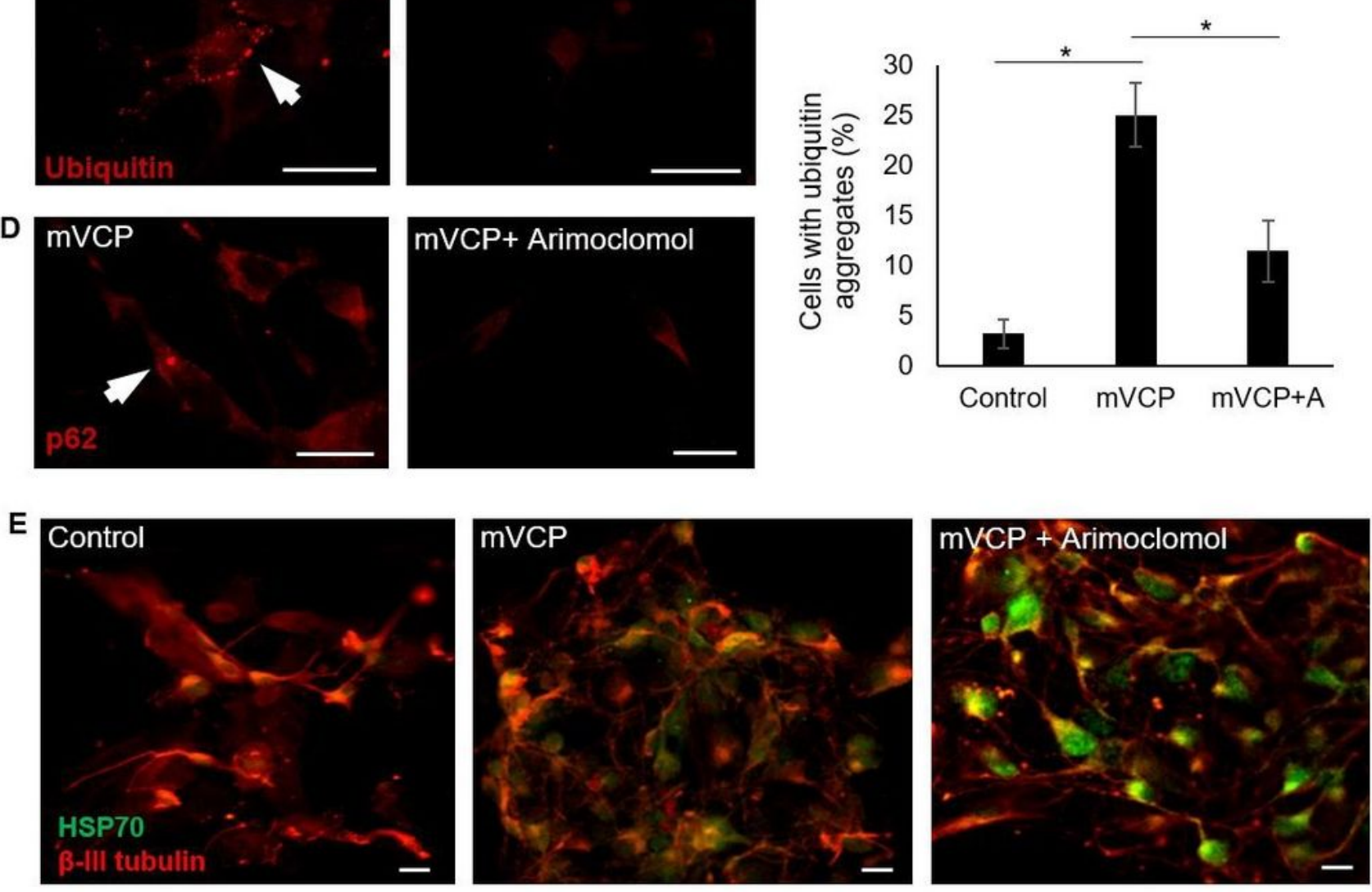


\section{Figure 8}

Human mVCP iPSC-derived motor neurons exhibit pathology ameliorated by Arimoclomol. (A) TDP-43 immunoreactivity shows localisation in control, mVCP and Arimoclomol treated mVCP iPSC motor neuron cultures. Inset shows magnification of cell with nuclear depletion of TDP-43 (B) Fluorescent images of ubiquitin immunoreactivity in MVCP iPSC motor neuron cultures with and without Arimoclomol treatment $(\mathrm{C})$ quantification of cells with ubiquitin-positive aggregates represented as a bar chart. * $\mathrm{p}=$ 0.026 between control and mVCP and $p=0.046$ between mVCP and mVCP+A. (D) p62 immunoreactivity in iPSC motor neurons from Arimoclomol treated and untreated mVCP cultures. (E) immunofluorescent images of HSP70 expression with neuronal marker $\beta$-III tubulin. DAPI labels nuclei (blue). Scale bar $=$ $20 \mu \mathrm{m}$.

\section{Supplementary Files}

This is a list of supplementary files associated with this preprint. Click to download.

- GreensmithSupplementaryMaterialNaturecomms2021.docx 